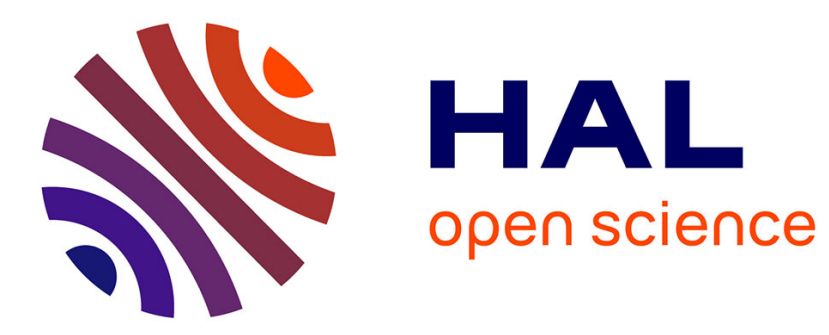

\title{
FRET-MC: a fluorescence melting competition assay for studying G4 structures in vitro
}

Yu Luo, Anton Granzhan, Daniela Verga, Jean-Louis Mergny

\section{To cite this version:}

Yu Luo, Anton Granzhan, Daniela Verga, Jean-Louis Mergny. FRET-MC: a fluorescence melting competition assay for studying G4 structures in vitro. Biopolymers, 2021, 112 (4), pp.e23415. 10.1002/bip.23415 . hal-03294475

\section{HAL Id: hal-03294475 \\ https://hal.science/hal-03294475}

Submitted on 21 Jul 2021

HAL is a multi-disciplinary open access archive for the deposit and dissemination of scientific research documents, whether they are published or not. The documents may come from teaching and research institutions in France or abroad, or from public or private research centers.
L'archive ouverte pluridisciplinaire HAL, est destinée au dépôt et à la diffusion de documents scientifiques de niveau recherche, publiés ou non, émanant des établissements d'enseignement et de recherche français ou étrangers, des laboratoires publics ou privés. 


\title{
FRET-MC: a fluorescence melting competition assay for
} studying G4 structures in vitro

\author{
Yu Luo $^{1,2}$, Anton Granzhan ${ }^{1}$, Daniela Verga ${ }^{1 *} \&$ Jean-Louis Mergny ${ }^{2 *}$ \\ 1. Université Paris Saclay, CNRS UMR9187, INSERM U1196, Institut Curie, 91400 \\ Orsay, France. \\ 2. Laboratoire d'Optique et Biosciences, Ecole Polytechnique, CNRS, Inserm, Institut \\ Polytechnique de Paris, 91128 Palaiseau, France. \\ * Authors to whom correspondence may be addressed: daniela.verga@curie.fr; jean- \\ louis.mergny@inserm.fr
}

Revised version, December 10, 2020

\section{Abstract}

G-quadruplexes (G4) play crucial roles in biology, analytical chemistry and nanotechnology.

The stability of G4 structures is impacted by the number of G-quartets, the length and positions of loops, flanking motifs, as well as additional structural elements such as bulges, capping base pairs, or triads. Algorithms such as G4Hunter or Quadparser may predict if a given sequence is G4-prone by calculating a quadruplex propensity score; however, experimental validation is still required. We previously demonstrated that this validation is not always straightforward, and that a combination of techniques is often required to unambiguously establish whether a sequence forms a G-quadruplex or not. In this article, we adapted the well-known FRETmelting assay to characterize G4 in batch, where the sequence to be tested is added, as an unlabeled competitor, to a system composed of a dual-labeled probe (F21T) and a specific quadruplex ligand. PhenDC3 was preferred over TMPyP4 because of its better selectivity for G-quadruplexes. In this so-called FRET-MC (melting competition) assay, G4-forming competitors lead to a marked decrease of the ligand-induced stabilization effect $\left(\Delta T_{\mathrm{m}}\right)$, while non-specific competitors (e.g., single- or double-stranded sequences) have little effect. Sixtyfive known sequences with different typical secondary structures were used to validate the assay, which was subsequently employed to assess eight novel sequences that were not previously characterized.

Keywords: G-quadruplex; FRET-melting; UV-melting; G-quartet; DNA structure 


\section{Introduction}

G-quadruplexes (G4) are four-stranded nucleic acid structures adopted by G-rich DNA and RNA sequences. G4 result from the stacking of two or more G-quartets (also called G-tetrads), which are formed by four guanine bases interacting through Hoogsteen hydrogen bonds ${ }^{1}$. G4 structures have been widely used to design biosensors to detect specific small molecules ${ }^{2-5}$ and to control the assembly of supramolecular DNA complexes ${ }^{6-9}$. G4 structures also exist in vivo and play important roles in cells ${ }^{10,11}$. For example, G4s can be formed at human telomeres and G4 ligands may interfere with telomeric functions, leading to telomere shortening and/or uncapping ${ }^{12-14}$. G4s are also found in the promoter regions of genes critical in cancer, including $K R A S, B C L 2$ and $V E G F^{15-17}$ : BCL2 plays an essential role in cell survival; VEGF is a key angiogenic growth factor which contributes to angiogenesis and tumor progression, and KRAS is one of the most frequently mutated oncogenes in many signal transduction pathways, relevant for different types of human carcinomas ${ }^{16}$.

Given the importance of G4 structures in biology and nanotechnology ${ }^{18}$, algorithms such as G4Hunter ${ }^{19,20}$ have been developed to predict if a specific sequence is G4-prone. However, for most DNA or RNA motifs, experimental validation is required and, for this purpose, a number of biophysical methods have been developed to characterize G4 structures in vitro. Some classical methods are based on the physical properties of G4 structures, such as UV-melting at $295 \mathrm{~nm}^{21}$, nuclear magnetic resonance (NMR) ${ }^{22}$, circular dichroism (CD) spectroscopy ${ }^{23}$, isothermal difference spectroscopy (IDS) and thermal difference spectroscopy (TDS) ${ }^{24}$. Fluorescence light-up assays employing dyes such as Thioflavin $\mathrm{T}^{25}, \mathrm{~N}$-methylmesoporphyrin IX (NMM) ${ }^{26}$, tailor-made dyes ${ }^{27}$ or combinations of dyes ${ }^{28,29}$ are also used to evidence G4 formation. Although there is a wide range of choices to check if a quadruplex is formed or not, this validation is not always straightforward; therefore, a combination of techniques is often required to unambiguously establish whether a sequence folds into a quadruplex or not ${ }^{19}$.

High-affinity G4 ligands can stabilize a G4 structure and alter its biological functions ${ }^{30}$. Typical assays used to characterize these ligands are the Fluorescent Intercalator Displacement (FID) assay ${ }^{31}$ and the fluorescence-based Förster Resonance Energy Transfer (FRET)-melting assay 
32, 33. The FRET-melting assay is based on the stabilization induced by a quadruplex ligand, leading to a difference in melting temperature $(\mathrm{Tm})$ between the nucleic acid alone and in presence of this ligand ${ }^{34}$ : in the presence of the latter, the thermal stability of the structure increases in a concentration-dependent manner. This FRET-melting assay has been extensively used to estimate whether a compound is a good quadruplex ligand or not ${ }^{34}$, with some biases when ranking ligands potency using melting experiments ${ }^{35}$. More recently, this assay was adapted to assess G4 ligands in near-physiological conditions ${ }^{36}$.

In this report, instead of testing unknown compounds, we make use of one of the mostcharacterized G4 ligands, PhenDC $3^{37}$, to determine if an unknown DNA sequence forms a Gquadruplex structure. PhenDC3 is a high affinity G4 ligand capable of binding to a variety of G4 structures, but with a low affinity for other conformations ${ }^{38,39}$. We took advantage of these observations to design a novel FRET-melting competition assay, termed FRET-MC, in which the interaction between a fluorescent G4-forming oligonucleotide and PhenDC3 is challenged by the (unlabeled) sequence of interest added in excess. Sixty-five sequences with a known structure were tested to validate this FRET-melting competition assay, which allowed us to determine whether a sequence forms a stable quadruplex or not. Finally, eight novel sequences were used to determine if this assay was accurate: the conclusions reached by this technique were supported by other biophysical methods (CD, IDS and TDS). Advantages and drawbacks of the FRET-MC method are discussed. 


\section{Materials and Methods}

\subsection{Samples}

Oligonucleotides were purchased from Eurogentec, Belgium, as dried samples: unmodified oligonucleotides were purified by RP cartridge while a dual-labeled F21T was purified by RPHPLC. Stock solutions were prepared at $100 \mu \mathrm{M}$ strand concentration for the unlabeled oligonucleotide and at $200 \mu \mathrm{M}$ strand concentration for F21T in $\mathrm{ddH}_{2} \mathrm{O} .50 \mu \mathrm{M}$ oligonucleotide solutions were annealed in the corresponding buffer, kept at $95{ }^{\circ} \mathrm{C}$ for $5 \mathrm{~min}$ and slowly cooled to room temperature before measurement. The FRET buffer contains $10 \mathrm{mM} \mathrm{KCl}, 90 \mathrm{mM} \mathrm{LiCl}$, $10 \mathrm{mM}$ lithium cacodylate, $\mathrm{pH}$ 7.2. The $\mathrm{K}-100$ buffer contains $100 \mathrm{mM} \mathrm{KCl,} 10 \mathrm{mM}$ lithium cacodylate, $\mathrm{pH} 7.2$.

\subsection{FRET-melting competition assay}

FRET melting experiments were performed in 96-well plates using a HT7900 RT-PCR instrument (Applied BioSystem). Each well contained competitors either at a single concentration $(3 \mu \mathrm{M})$, or at 6 different concentrations, ranging from 0.2 to $3 \mu \mathrm{M}$. $0.2 \mu \mathrm{M}$ of the fluorescent oligonucleotide F21T was incubated with or without $0.4 \mu \mathrm{M} \mathrm{G4}$ ligand (PhenDC3 for most experiments; TMPyP4 was used in a control experiment shown in supplementary information) in FRET buffer in a final volume of $25 \mu \mathrm{L}$. The FAM channel was used to collect the fluorescence signal. Samples were kept at $25^{\circ} \mathrm{C}$ for $5 \mathrm{~min}$, then the temperature was increased by $0.5^{\circ} \mathrm{C}$ per minute until $95^{\circ} \mathrm{C}$. Each experimental condition was tested in duplicate on at least two separate plates.

$\Delta \mathrm{Tm}$ is determined as the difference in $\mathrm{Tm}$ with the sample containing F21T in the absence of PhenDC3. The Tm of an oligonucleotide is defined as the temperature at which $50 \%$ of the oligonucleotide is unfolded. The most common method to obtain Tm values is approximated as corresponding to half of the height at the normalized melting curve ${ }^{40}$.

The FRET-melting assay uses a 96-well plate as a sample holder, which allows to process 48 sequences simultaneously. The traditional 'midpoint' determination requires a manual analysis

${ }^{41}$. Given the number of profiles to be analyzed, this process is time consuming as the curves 
are analyzed one by one. The DoseResp Function in Origin Pro package allows collect Tm in batch. As shown in Fig. S1A, $\log \mathrm{X}_{0}$ in fitting curve is the Tm. This method can only be used in curve (i) (see results) as non-linear fitting would fail if there is no high plateau at row curve. In general, the difference of Tm calculated by these two methods is very small: when using both the ' $1 / 2$ height' and non-linear fitting methods to calculate Tm of F21T alone, the results are $59.8^{\circ} \mathrm{C}$ and $59.4^{\circ} \mathrm{C}$, respectively (Fig. S1B). In a few rare cases that should be noted (Fig. S1C), curves are irregular, and the minimum of $\mathrm{X}$-axis (usually $25^{\circ} \mathrm{C}$ in FRET-melting assay) does not correspond to $\mathrm{y}=0$ for the normalized curve. In this case, using ' $1 / 2$ height' is more accurate; Tm calculated by DoseResp always referenced the $\mathrm{X}$ value corresponding to $\mathrm{Y}=0.5$, while in some curves the $1 / 2$ height $\left(\mathrm{Y}_{\mathrm{x}=95}-\mathrm{Y}_{\mathrm{x}=25}\right)$ at $\mathrm{Y}$-axis is not always 0.5 . In the instance, $1 / 2$ height at $\mathrm{Y}$-axis is 0.525 , Tm calculated by ' $1 / 2$ height' is $53.0^{\circ} \mathrm{C}$, to be compared with $51.8^{\circ} \mathrm{C}$ by DoseResp.

In brief, DoseResp is only truly accurate for curves with appropriate upper and lower baselines, while the ' $1 / 2$ height' approach can be applied in all cases, provided that normalization is accurate. If possible, we suggest to use the same method to calculate $\mathrm{Tm}$ for experiments performed in parallel, although differences of Tm determined by these two approaches is small in general.

\subsection{UV-melting assay}

UV-melting curves of $5 \mu \mathrm{M}$ oligonucleotides in FRET buffer were recorded with a Cary 300 (Agilent Technologies, France) spectrophotometer. Heating runs were performed between $10^{\circ} \mathrm{C}$ and $95{ }^{\circ} \mathrm{C}$, the temperature was increased by $0.2{ }^{\circ} \mathrm{C}$ per minute, and absorbance was recorded at $260 \mathrm{~nm}$ and $295 \mathrm{~nm}$. Tm was determined as the temperature corresponding to half of the height of the normalized melting curve. 
$1313 \mu \mathrm{M}$ SP-PGQ-1 was kept in $1000 \mu \mathrm{L}$ FRET buffer. $3 \mu \mathrm{M}$ Oligonucleotides of testing set were 132 kept in $1000 \mu \mathrm{L} \mathrm{K-100} \mathrm{buffer.} \mathrm{CD} \mathrm{spectra} \mathrm{were} \mathrm{recorded} \mathrm{on} \mathrm{a} \mathrm{JASCO} \mathrm{J-1500} \mathrm{(France)}$ 133 spectropolarimeter at room temperature, using a scan range of 400-230 nm, a scan rate of 200 $134 \mathrm{~nm} / \mathrm{min}$ and averaging four accumulations.

\subsection{Thermal difference spectra}

$3 \mu \mathrm{M}$ SP-PGQ-1 was kept in FRET buffer. $3 \mu \mathrm{M}$ Oligonucleotides of testing set were kept in $1000 \mu \mathrm{L}$ K-100 buffer. Absorbance spectra were recorded on a Cary 300 (Agilent Technologies, France) spectrophotometer at $25^{\circ} \mathrm{C}$ (scan range: $500-200 \mathrm{~nm}$; scan rate: $600 \mathrm{~nm} / \mathrm{min}$; automatic baseline correction). After recording the first spectra (folded), temperature was increased to $95{ }^{\circ} \mathrm{C}$, and the second UV-absorbance spectra was recorded after 15 min of equilibration at high temperature. TDS corresponds to the arithmetic difference between the initial (folded; $25^{\circ} \mathrm{C}$ ) and second (unfolded; $95^{\circ} \mathrm{C}$ ) spectra.

\subsection{Isothermal difference spectra}

$1443 \mu \mathrm{M}$ Oligonucleotides of testing set were kept in $900 \mu \mathrm{L}$ K-100 buffer. Absorbance spectra

145 were recorded on a Cary 300 (Agilent Technologies, France) spectrophotometer at $25^{\circ} \mathrm{C}$ (scan range: $500-200 \mathrm{~nm}$; scan rate: $600 \mathrm{~nm} / \mathrm{min}$; automatic baseline correction). $100 \mu \mathrm{L}$ of $1 \mathrm{M} \mathrm{KCl}$

147 was added after recording the first spectrum, and the second UV-absorbance spectrum was recorded after 15 min of equilibration. IDS correspond to the arithmetic difference between the

149 initial (unfolded) and second (folded, thanks to the addition of $\mathrm{K}^{+}$) spectra, after correction for 150 dilution. 


\section{Results and Discussion}

153

154

155

156

157

158

159

160

161

162

163

164

165

166

167

168

169

170

171

172

173

174

175

176

\subsection{Principle of the FRET-MC assay}

In a $\mathrm{K}^{+}$-containing buffer, F21T forms a stable $\mathrm{G} 4$ structure which can be used as a FRETmelting probe thanks to the fluorophores attached at both ends: a 5'-appended donor (Fluorescein) and a 3'-appended acceptor (TAMRA), allowing efficient energy transfer between the donor and acceptor dyes when the oligonucleotide is folded (Fig. 1). Thermal unfolding leads to the disruption of the G-quadruplex structure and a decrease in FRET efficiency, as the 5' and 3' ends become distant when the sequence is single-stranded. In the presence of a G4 ligand, the melting temperature $\left(T_{\mathrm{m}}\right)$ of F21T increases, as the ligand makes the structure of F21T more stable. F21T is typically used in a FRET-melting assay, in which the specificity of a ligand is tested by adding various specific (G4-forming) and unspecific (duplexes or single-stranded) competitors ${ }^{33}$. In this report, we are radically changing our viewpoint: rather than testing ligands of unknown specificity against known competitors, we are investigating one of the best-characterized G4-ligand, PhenDC3, against a variety of oligonucleotide competitors. Adding a large excess of an unlabeled oligonucleotide may lead to two possible scenarios:

(i) The competitor is unable to trap the quadruplex ligand. In this case, Tm of the (F21T + PhenDC3) system is not affected by the competing oligonucleotide (in other words, $\Delta \mathrm{Tm}$ remains high). This is the expected outcome for a single-strand or a DNA or RNA duplex.

(ii) If the unlabeled competitor has a high affinity for PhenDC3, it will sequester a significant fraction of the compound, which will be no longer available for F21T stabilization, leading to a decrease in Tm. In this case, if the competition is very efficient, the Tm should fall back close to the value obtained without quadruplex ligand, meaning for $\mathrm{F} 21 \mathrm{~T}$ alone (in other words, $\Delta \mathrm{Tm} \approx 0$ ).

177 To proceed, we selected a variety of competitors for which the structure was previously

178 investigated and characterized ${ }^{42}$. This collection of over 60 sequences includes a variety of 


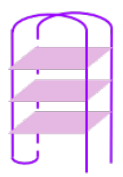

G4 Structure
A
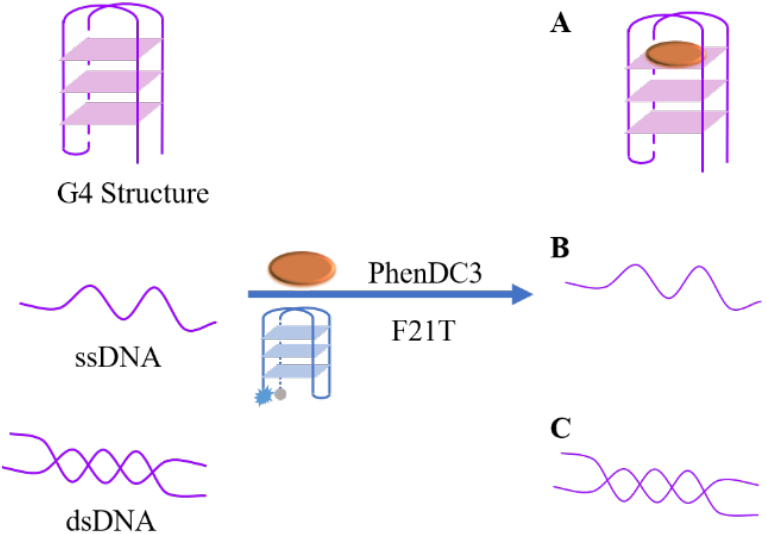

DDNA
C

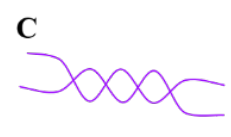

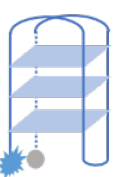

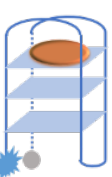

Heating

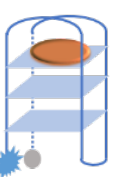

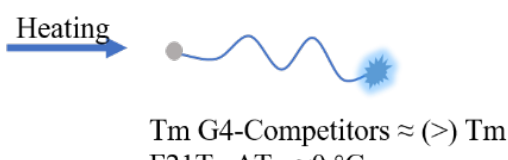
F21T; $\Delta \mathrm{Tm} \approx 0{ }^{\circ} \mathrm{C}$.
182

183

184

185

3.2 Validation of the FRET-melting competition assay with a set of 65 sequences.

A trivial, but important, control was first performed by checking that the competitors do not directly interact with F21T. To that aim, Tm of F21T was measured alone or in the presence of each competitor, in the absence of PhenDC3. As expected, most sequences tested had negligible, if any, effect on F21T melting (Fig. S2; normalized FRET-melting curves are shown in Fig. S3). A few motifs (46AG, T95-2T, T2B-1, AT11, LWDLN1, AND1, RND3, RND6 and AT26) led to a significant decrease in $\operatorname{Tm}\left(\Delta \mathrm{Tm}>5^{\circ} \mathrm{C}\right)$.

We next investigated the impact of the competitors on the stabilization effect $(\Delta \mathrm{Tm})$ induced by PhenDC3 on F21T. Fig. 2 presents examples of FRET-melting profiles for F21T alone (Fig. 
A

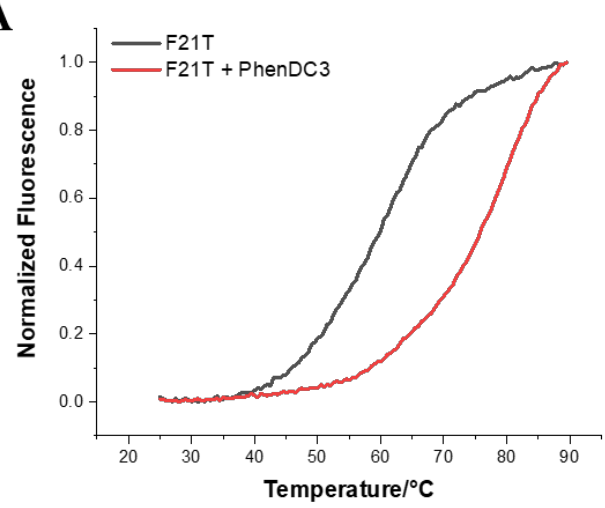

B

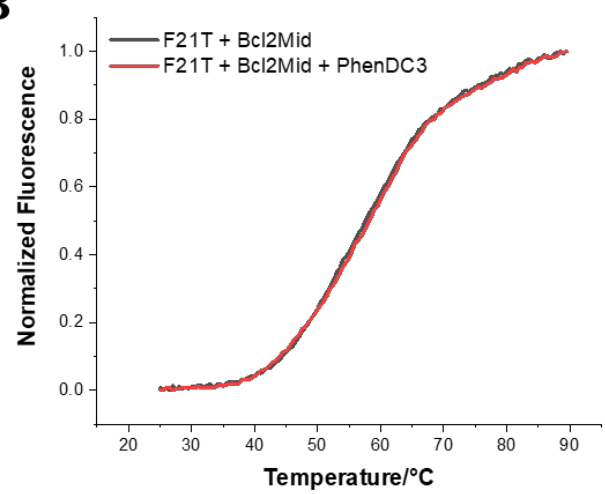

$\mathbf{C}$

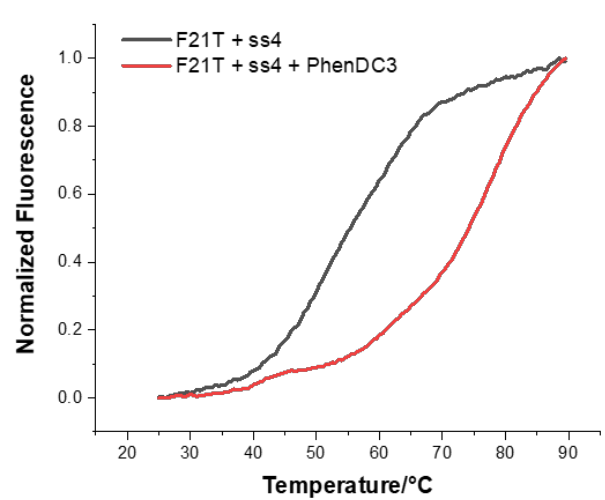

D

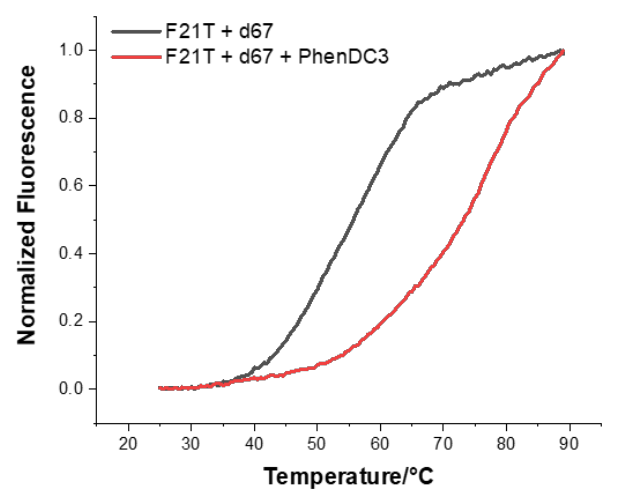

Fig. 2. FRET-melting profiles of $0.2 \mu \mathrm{M} \mathrm{F21T}$ alone or in the presence of $3 \boldsymbol{\mu M}$ competitors, and with (red) or without (black) $0.4 \mu M$ PhenDC3. (A) F21T alone and in the presence of PhenDC3, and with the addition of the competitor: (B) G4 structure, (C) single-strand DNA and (D) duplex DNA. Samples were annealed and measured in $10 \mathrm{mM} \mathrm{KCl}, 90 \mathrm{mM} \mathrm{LiCl}, 10$ $\mathrm{mM}$ lithium cacodylate $\mathrm{pH} 7.2$ buffer.

In the absence of any competitor, 2 eqv. of PhenDC 3 induces a $\Delta \mathrm{Tm}$ of $23.4^{\circ} \mathrm{C}$, in agreement with previous results ${ }^{43}$. As expected, none of the competitors induced a further significant increase in Tm, as compared to F21T + PhenDC3. Fig. 3 summarizes the $\Delta$ Tm results obtained for F21T in the presence of PhenDC3 and in the presence or absence of oligonucleotide competitor (normalized FRET-melting curves shown in Fig. S3). Many, but not all, of the sequences known to form G4 structures led to a significant drop in $\Delta \mathrm{Tm}$ values (upper half of the figure), showing that they acted as efficient competitors. In contrast, single-stranded and 
212 duplex DNAs had little impact, as $\Delta \mathrm{Tm}$ values remained high, close to the value found for F21T

213 + PhenDC3 with no competitor. To quantitate this competition effect, we defined the S Factor,

214 as originally described in ${ }^{44}$, which corresponds to the relative PhenDC3 stabilization remaining

215 in the presence of the competitor:

$$
S \text { Factor }=\frac{\Delta \mathrm{Tm} \text { of F21T with competitors }}{\Delta \mathrm{Tm} \text { of F21T alone }}
$$

217 Based on S Factor, the competitors can be divided into two categories: (i) ineffective 218 competitors, for which $S$ remains $\approx 1$, meaning that competition is nearly completely 219 unproductive, as expected for a structure for which PhenDC3 has no affinity, and conversely 220 (ii) potent competitors (i.e., stable quadruplexes) would give a $S$ Factor close to 0 (Fig. 3). 


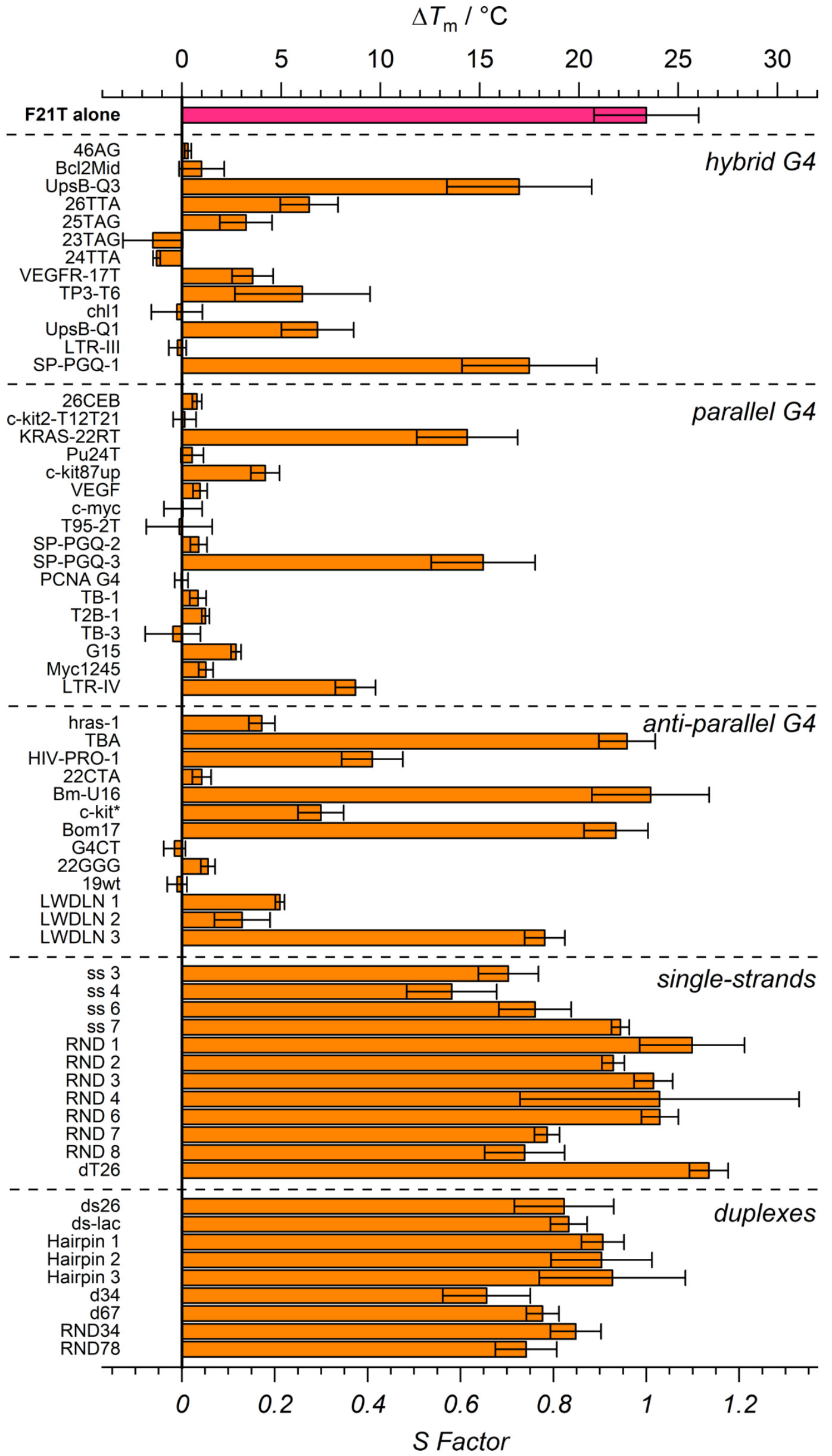



$\boldsymbol{\mu M}$ competitors. The $S$ Factor (bottom $\mathrm{X}$-axis) provides a normalized value. Samples were annealed and measured in $10 \mathrm{mM} \mathrm{KCl}, 90 \mathrm{mM} \mathrm{LiCl}, 10 \mathrm{mM}$ lithium cacodylate $\mathrm{pH} 7.2$ buffer.

As expected, the majority of G4-forming sequences led to $S$ values close to 0 . However, several known G4 structures (UpsB-Q3, SP-PGQ-1, KRAS-22RT, SP-PGQ-3, TBA, Bm-U16, Bom17 and LWDLN3) were not efficient competitors $(S>0.6)$. In order to understand these results, we performed UV-melting experiments for these 8 sequences and collected Tm values in Table S2 (detailed UV-melting curves are shown in Fig. S4). The thermal stability of all these sequences (except for SP-PGQ-1) was relatively low, indicating they form unstable G4 structures, which are likely to be unfolded in the temperature range where F21T starts to melt: they are then "seen" as non-specific single-strands rather than true G-quadruplexes. SP-PGQ1 behaved differently: UV-absorbance at $295 \mathrm{~nm}$ of a quadruplex should decrease upon heating due to the unfolding of the quadruplex structure. Although SP-PGQ-1 was reported to form a hybrid G4 structure ${ }^{45}$, our results show an unexpected increase in absorbance at $295 \mathrm{~nm}$ upon UV-melting, incompatible with the unfolding of a quadruplex, and rather suggesting the formation of a another structure (e.g., a mismatched duplex) at low temperatures. CD spectroscopy and TDS confirmed this hypothesis (Fig. S5). In contrast, the Tm of 10 different G4 sequences acting as effective competitors ( $S<0.3$ for 46AG, Bcl2Mid, 25TGA, Chl, LTRIII, c-kit-T12T2, Pu24T, c-kit87up, VEGF and T95-2T) were always higher than the Tm of the false negative sequences, as shown in Table S2 (UV-melting curves showed in Fig. S4). Although some of the Tm are still lower than the Tm of F21T, their presence in large excess (15-fold molar excess as compared to F21T) may compensate for a partial denaturation.

We then investigated whether one could substitute PhenDC3 by a different G4 ligand, TMPyP4, a cationic porphyrin which also has a high affinity for G-quadruplexes, but is much less selective. Fig. S6 presents $\Delta \mathrm{Tm} / S$ values for $0.4 \mu \mathrm{M}$ TMPyP4 on $0.2 \mu \mathrm{M}$ F21T, alone or in

247 the presence of various competitors at $3 \mu \mathrm{M}$ strand concentration. Experiments were done in a 248 buffer identical to the one used for PhenDC3. We tested 5 representative sequences for each structural type considered here (25 different competitors in total). Although parallel 
quadruplexes were the most efficient competitors, some single-strands and most duplexes were also competing, with $S$ value around 0.5, lower than the $S$ values found for most anti-parallel and two hybrid quadruplexes with TMPyP4. This experiment demonstrates that, for this method to be reliable, a truly specific G4 ligand has no preferential binding to any G4 topology must be chosen. While other compounds than PhenDC3 may fit the bill, moderately selective compounds will not.

A critical factor for the competition efficiency (measured by the $S$ value) in the FRET-MC assay should be the affinity and number of binding sites present on the competitor sequence for the quadruplex ligand (PhenDC3 in all further experiments). We wanted to investigate if the thermal stability of the structure itself would also contribute. All duplex sequences tested here had a significantly higher Tm value than F21T (as shown in Table S2); they were still poor competitors, even when added in large excess, as PhenDC3 is unable to bind to duplexes.

For the quadruplexes, in order to investigate how $S$ value correlates with Tm, we tried a range of 6 different competitor concentrations ( $1 \mathrm{x}$ to $30 \mathrm{x}$ molar excess, as compared to $\mathrm{F} 21 \mathrm{~T}$ ) for 9 different G4-forming sequences. As shown in Fig. S7, the Tm for each quadruplex (detailed UV-melting curves are shown in Fig. S4) was experimentally determined under identical conditions, and is shown in red below; sequences were ranked from left (lowest Tm) to right (highest Tm). Overall, there is indeed a correlation between $\mathrm{Tm}$ and $S$ values: the competitors with a high thermal stability lead to low $S$ values when the competitor is not in huge excess. Since $S$ values are low for nearly all sequences at 3 or $6 \mu \mathrm{M}$, independently of Tm, we did not consider these two concentrations in Fig. S8, which provides a different view / representation of these $S$ values, in which sequences are clustered in three categories according to Tm. The most striking difference is found between the high stability group and the others. Table S3 summarizes average $S$ values (from 1x to 10x molar excess) for these 9 sequences. Generally, average $S$ values decreased with increasing Tm but the correlation is far from perfect. The average $S$ values for high stability sequences are 0.25 or below, while $S$ values for the others are above 0.35 . 
278 To test this method on a set of novel sequences, we used eight genomic DNA sequences of 279 unknown structure. These motifs are found close to the transcription start site of different 280 human promoters, and their biological relevance is currently being investigated. According to 281 G4Hunter analysis ${ }^{19}$, four of them (UN1-4) were likely to form a quadruplex structure, as their 282 G4Hunter score was above 1.2, while the remaining four (UN5-8) were unlikely to adopt a G4 283 conformation, with a G4Hunter score $<1.0$. Detailed sequences with location information 284 (Human hg19) and G4Hunter scores are shown in Table S4.

285 The $\Delta \mathrm{Tm}$ values and $S$-factors for the tested sequences are shown on Fig. 4. The first four 286 oligonucleotides (UN1-4) with high G4-hunter scores gave low S-factor values $(<0.1)$, 287 consistent with G4 formation. In contrast, the remaining four samples (UN5-8) were poor 288 competitors $(S>0.8)$ and unlikely to form quadruplexes, in agreement with their low G4Hunter 289 scores. These conclusions were confirmed by three independent techniques (CD, IDS and TDS) 290 measured in $100 \mathrm{mM} \mathrm{KCl} \mathrm{(Fig} \mathrm{S9),} \mathrm{all} \mathrm{giving} \mathrm{results} \mathrm{consistent} \mathrm{with} \mathrm{the} \mathrm{formation} \mathrm{of} \mathrm{G4}$ 291 structures by UN1-4 and the absence of these structures in the case of UN5-8. 

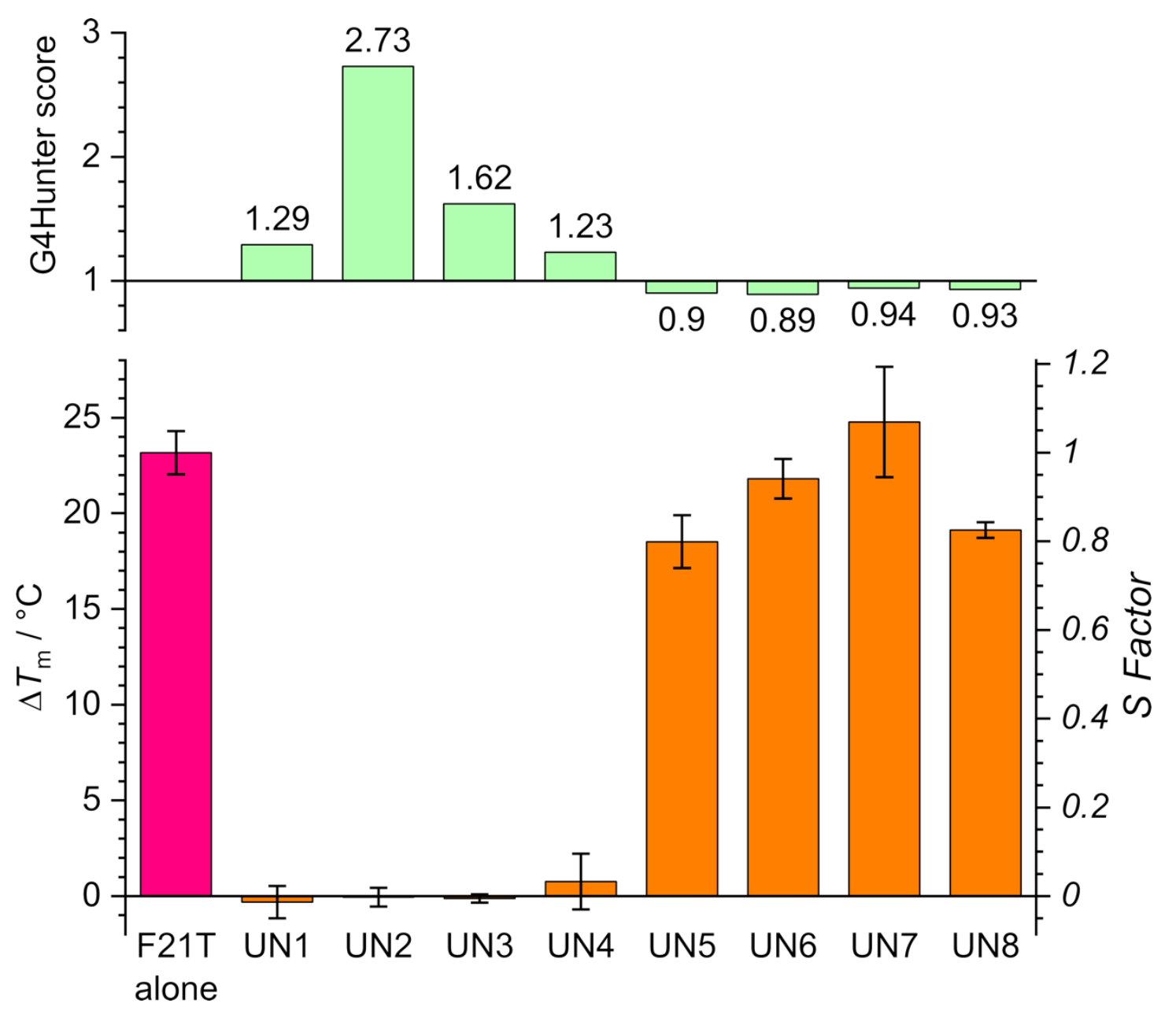

Fig. 4. Testing 8 different sequences (UN1-UN8) with the FRET-MC assay. The G4Hunter score for each competitor is indicated on the upper part of the figure (green bars). The $\Delta \mathrm{Tm}$ induced by $0.4 \mu \mathrm{M}$ PhenDC 3 on $0.2 \mu \mathrm{M}$ F21T, alone or in the presence of $3 \mu \mathrm{M}$ competitors (UN1-8) is plotted on the lower part of the graph. The $S$ Factor (right Y-axis) provides a normalized value. Samples were annealed and measured in $10 \mathrm{mM} \mathrm{KCl}, 90 \mathrm{mM} \mathrm{LiCl}, 10 \mathrm{mM}$ lithium cacodylate $\mathrm{pH} 7.2$ buffer.

\section{Conclusion}

The FRET-MC assay described here is a fast and inexpensive characterization method to determine if a sequence is forming a stable quadruplex or not. It offers several advantages: expensive, minimal amounts are used for each point as the volume is reduced $(25 \mu \mathrm{L})$ 
and its concentration is only $0.2 \mu \mathrm{M}$, and can even be further reduced if necessary, provided a sensitive RT-PCR instrument is available. Conversely, the assayed sequences are unmodified oligonucleotides and do not require extensive purification; only 25 picomoles are needed per point.

- It is fast: FRET-melting takes 1-2 hours while UV-melting requires several hours (14 $\mathrm{h}$ with the temperature gradient used here) for each experiment.

- It is simple to set up: all reagents are commercially available (PhenDC3 included) and the FRET melting assay is now routinely used in a number of labs.

- It allows testing multiple samples in parallel. While a classical UV spectrophotometer can only read up to 6 or 9 samples, FRET-melting uses a 96-well plate as a sample holder, and it is able to process 48 sequences in duplicate. It may even be transposed to 384-well format.

At the same time, this method has several limitations:

- The main assumptions for this technique are that PhenDC3 $i$ ) indiscriminately binds to all Gquadruplexes, and ii) does not bind to other structures. In other words, for this method to work, we need a perfect, general G4 ligand with high structure specificity. Previously published studies ${ }^{31,37}$ have shown that PhenDC 3 does indeed bind to all G4 tested so far, and has excellent specificity. We cannot exclude, however, that PhenDC3 would also recognize other unusual motifs such as G-triplexes ${ }^{46}$. Additional experiments are therefore required to reach a clear conclusion for a given sequence: as previously stated ${ }^{19}$, we advocate the use of several independent techniques to assess G4 formation. On the other hand, a high-affinity but poorly selective quadruplex ligand such as TMPyP4 proved to be ineffective for this application.

- The sequence to be tested should not be complementary to F21T, as it would interfere with the structure of the fluorescent probe. C-rich sequences, and especially repetitions of the CCCTAA hexanucleotide motif should be avoided. The training set chosen for this study did not involve any i-motif sequence, resulting from the folding of C-rich oligonucleotides. These sequences would then be partially complementarity to the fluorescent G-rich oligonucleotide F21T: duplex formation would "kill" the assay by interfering with G4 formation. On the other 
hand, the method itself may later be adapted to the analysis of i-motif sequences. This assay would involve a "complementary" sequence for i-motif formation, in which the fluorescently

335 labeled oligonucleotide is C-rich, not G-rich, and forms an i-motif itself. But this would require a "perfect" i-motif ligand as well, i.e. a compound that would bind reasonably well to all imotifs while having no affinity for any other structure. The i-motif ligands we have tested do not meet these criteria, and cannot be considered as equivalent to PhenDC3 for this purpose.

- The main limitation of this FRET-melting competition assay is its inability to detect unstable quadruplexes which behave as single-strands (Fig. 3) at the temperature where F21T starts to melt. This assay should therefore be employed to identify moderately stable or highly stable

342 G4 structures. A possible way to circumvent this limitation would be to replace or complement 343 F21T by a quadruplex probe with lower stability ${ }^{47}$. A two-quartet quadruplex such as the 344 thrombin binding aptamer (TBA) could be proposed, keeping in mind that these G4 are often 345 weaker binders for G4 ligands such as PhenDC3. In any case, unstable quadruplexes are less 346 likely to be biologically relevant ${ }^{48}$ and unlikely to be identified by genome-wide methods such 347 as G4-seq ${ }^{49}$ as extension is performed at $60^{\circ} \mathrm{C}$ during Illumina sequencing.

348 - Finally, the $S$ value cannot be used as a proxy for the (thermal) stability of the tested 349 quadruplex. While there is some correlation between thermal stability and competition 350 efficiency (stable G4 tend to give lower $S$ values), other factors contribute to the competition 351 efficiency, such as the affinity of the PhenDC3 ligand for this topology, and the number of 352 binding sites available.

353 Overall, despites the shortcomings listed above, the FRET-melting competition assay should 354 constitute an interesting addition to the in vitro "G4 characterization toolbox". 
356 Raw data / melting profiles may be downloaded at

357 https://data.mendeley.com/datasets/gspc9r73r5/1.

358

359

Acknowledgments

360 We thank both reviewers for excellent suggestions, Laurent Lacroix (ENS, Paris) for helpful 361 discussions, and Corinne Landras Guetta and Marie-Paule Teulade-Fichou (Institut Curie, 362 Orsay) for a sample of PhenDC3. This manuscript is dedicated to the memory of Prof. Michael 363 J. Waring, with whom J.L.M. had interesting lively discussions about DNA ligands during his 364 sabbatical in France.

365

366 Conflict of interest

367 None 
370 1. Gellert, M.; Lipsett, M. N.; Davies, D. R., Helix formation by guanylic acid. Proc Natl 371 Acad Sci U S A 1962, 48 (12), 2013-8.

372 2. Guo, Y.; Xu, L.; Hong, S.; Sun, Q.; Yao, W.; Pei, R., Label-free DNA-based 373 biosensors using structure-selective light-up dyes. Analyst 2016, 141 (24), 6481-6489.

374 3. He, H. Z.; Chan, D. S.; Leung, C. H.; Ma, D. L., G-quadruplexes for luminescent 375 sensing and logic gates. Nucleic Acids Res 2013, 41 (8), 4345-59.

376 4. Ren, J.; Wang, T.; Wang, E.; Wang, J., Versatile G-quadruplex-mediated strategies in 377 label-free biosensors and logic systems. Analyst 2015, 140 (8), 2556-72.

378 5. Ruttkay-Nedecky, B.; Kudr, J.; Nejdl, L.; Maskova, D.; Kizek, R.; Adam, V., G379 quadruplexes as sensing probes. Molecules 2013, 18 (12), 14760-79.

380 6. Lustgarten, O.; Carmieli, R.; Motiei, L.; Margulies, D., A Molecular Secret Sharing 381 Scheme. Angew Chem Int Ed Engl 2019, 58 (1), 184-188.

382 7. Mergny, J. L.; Sen, D., Correction to DNA Quadruple Helices in Nanotechnology. Chem 383 Rev 2020.

8. Stefan, L.; Monchaud, D., Applications of guanine quartets in nanotechnology and chemical biology. Nature Reviews Chemistry 2019, 3 (11), 650-668.

9. Cao, Y.; Gao, S.; Yan, Y.; Bruist, M. F.; Wang, B.; Guo, X., Assembly of supramolecular DNA complexes containing both G-quadruplexes and i-motifs by enhancing the G-repeat-bearing capacity of i-motifs. Nucleic Acids Res 2017, 45 (1), 26-38.

10. Spiegel, J.; Adhikari, S.; Balasubramanian, S., The Structure and Function of DNA GQuadruplexes. Trends Chem 2020, 2 (2), 123-136.

11. Varshney, D.; Spiegel, J.; Zyner, K.; Tannahill, D.; Balasubramanian, S., The regulation and functions of DNA and RNA G-quadruplexes. Nat Rev Mol Cell Biol 2020, 21 393 (8), 459-474.

12. Burger, A. M.; Dai, F.; Schultes, C. M.; Reszka, A. P.; Moore, M. J.; Double, J. A.; Neidle, S., The G-Quadruplex-Interactive Molecule BRACO-19 Inhibits Tumor Growth, Consistent with Telomere Targeting and Interference with Telomerase Function. Cancer Research 2005, 65 (4), 1489.

13. Read, M.; Harrison, R. J.; Romagnoli, B.; Tanious, F. A.; Gowan, S. H.; Reszka, A. P.; Wilson, W. D.; Kelland, L. R.; Neidle, S., Structure-based design of selective and potent G quadruplex-mediated telomerase inhibitors. Proceedings of the National Academy of Sciences 2001, 98 (9), 4844.

14. Riou, J. F.; Guittat, L.; Mailliet, P.; Laoui, A.; Renou, E.; Petitgenet, O.; Mégnin-Chanet, F.; Hélène, C.; Mergny, J. L., Cell senescence and telomere shortening 404 induced by a new series of specific G-quadruplex DNA ligands. Proceedings of the National 405 Academy of Sciences USA 2002, 99 (5), 2672. 
15. Brooks, T. A.; Kendrick, S.; Hurley, L., Making sense of G-quadruplex and i-motif functions in oncogene promoters. FEBS J 2010, 277 (17), 3459-69.

16. Jana, J.; Mondal, S.; Bhattacharjee, P.; Sengupta, P.; Roychowdhury, T.; Saha, P.; Kundu, P.; Chatterjee, S., Chelerythrine down regulates expression of VEGFA, BCL2 and KRAS by arresting G-Quadruplex structures at their promoter regions. Sci Rep 2017, 7, 40706.

17. Marquevielle, J.; Robert, C.; Lagrabette, O.; Wahid, M.; Bourdoncle, A.; Xodo, L. E.; Mergny, J. L.; Salgado, G. F., Structure of two G-quadruplexes in equilibrium in the KRAS promoter. Nucleic Acids Res 2020, 48 (16), 9336-9345.

18. Spiegel, J.; Adhikari, S.; Balasubramanian, S., The Structure and Function of DNA GQuadruplexes. Trends in Chemistry 2020, 2 (2), 123-136.

19. Bedrat, A.; Lacroix, L.; Mergny, J. L., Re-evaluation of G-quadruplex propensity with G4Hunter. Nucleic Acids Res 2016, 44 (4), 1746-59.

20. Brazda, V.; Kolomaznik, J.; Lysek, J.; Bartas, M.; Fojta, M.; Stastny, J.; Mergny, J. L., G4Hunter web application: a web server for G-quadruplex prediction. Bioinformatics 2019, 35 (18), 3493-3495.

21. Alba, J. J.; Sadurni, A.; Gargallo, R., Nucleic Acid i-Motif Structures in Analytical Chemistry. Crit Rev Anal Chem 2016, 46 (5), 443-54.

22. Adrian, M.; Heddi, B.; Phan, A. T., NMR spectroscopy of G-quadruplexes. Methods 2012, 57 (1), 11-24.

23. Kypr, J.; Kejnovska, I.; Renciuk, D.; Vorlickova, M., Circular dichroism and conformational polymorphism of DNA. Nucleic Acids Res 2009, 37 (6), 1713-25.

24. Mergny, J. L.; Li, J.; Lacroix, L.; Amrane, S.; Chaires, J. B., Thermal difference spectra: a specific signature for nucleic acid structures. Nucleic Acids Res 2005, 33 (16), e138.

25. Renaud de la Faverie, A.; Guedin, A.; Bedrat, A.; Yatsunyk, L. A.; Mergny, J. L., Thioflavin T as a fluorescence light-up probe for G4 formation. Nucleic Acids Res 2014, 42 (8), e65.

26. Sabharwal, N. C.; Savikhin, V.; Turek-Herman, J. R.; Nicoludis, J. M.; Szalai, V. A.; Yatsunyk, L. A., N-methylmesoporphyrin IX fluorescence as a reporter of strand orientation in guanine quadruplexes. FEBS J 2014, 281 (7), 1726-37.

27. Xie, X.; Renvoisé, A.; Granzhan, A.; Teulade-Fichou, M.-P., Aggregating distyrylpyridinium dye as a bimodal structural probe for G-quadruplex DNA. New Journal of Chemistry 2015, 39 (8), 5931-5935.

28. Kreig, A.; Calvert, J.; Sanoica, J.; Cullum, E.; Tipanna, R.; Myong, S., Gquadruplex formation in double strand DNA probed by NMM and CV fluorescence. Nucleic Acids Res 2015, 43 (16), 7961-70.

29. Zuffo, M.; Xie, X.; Granzhan, A., Strength in Numbers: Development of a Fluorescence Sensor Array for Secondary Structures of DNA. Chemistry 2019, 25 (7), 1812-1818. 
30. O'Hagan, M. P.; Morales, J. C.; Galan, M. C., Binding and Beyond: What Else Can GQuadruplex Ligands Do? European Journal of Organic Chemistry 2019, 2019 (31-32), 49955017.

31. Tran, P. L.; Largy, E.; Hamon, F.; Teulade-Fichou, M. P.; Mergny, J. L., Fluorescence intercalator displacement assay for screening G4 ligands towards a variety of Gquadruplex structures. Biochimie 2011, 93 (8), 1288-96.

32. De Cian, A.; Guittat, L.; Kaiser, M.; Sacca, B.; Amrane, S.; Bourdoncle, A.; Alberti, P.; Teulade-Fichou, M. P.; Lacroix, L.; Mergny, J. L., Fluorescence-based melting assays for studying quadruplex ligands. Methods 2007, 42 (2), 183-95.

33. Mergny, J.-L.; Maurizot, J.-C., Fluorescence Resonance Energy Transfer as a Probe for GQuartet Formation by a Telomeric Repeat. ChemBioChem 2001, 2 (2), 124-132.

34. Renciuk, D.; Zhou, J.; Beaurepaire, L.; Guedin, A.; Bourdoncle, A.; Mergny, J. L., A FRET-based screening assay for nucleic acid ligands. Methods 2012, 57 (1), 122-8.

35. Marchand, A.; Rosu, F.; Zenobi, R.; Gabelica, V., Thermal Denaturation of DNA GQuadruplexes and Their Complexes with Ligands: Thermodynamic Analysis of the Multiple States Revealed by Mass Spectrometry. J Am Chem Soc 2018, 140 (39), 12553-12565.

36. Morgan, R. K.; Psaras, A. M.; Lassiter, Q.; Raymer, K.; Brooks, T. A., G-quadruplex deconvolution with physiological mimicry enhances primary screening: Optimizing the FRET Melt(2) assay. Biochim Biophys Acta Gene Regul Mech 2020, 1863 (1), 194478.

37. De Cian, A.; DeLemos, E.; Mergny, J.-L.; Teulade-Fichou, M.-P.; Monchaud, D., Highly Efficient G-Quadruplex Recognition by Bisquinolinium Compounds. Journal of the American Chemical Society 2007, 129 (7), 1856-1857.

38. Marchand, A.; Granzhan, A.; Iida, K.; Tsushima, Y.; Ma, Y.; Nagasawa, K.; Teulade-Fichou, M. P.; Gabelica, V., Ligand-induced conformational changes with cation ejection upon binding to human telomeric DNA G-quadruplexes. $J$ Am Chem Soc 2015, 137 (2), 750-6.

39. Ruggiero, E.; Richter, S. N., G-quadruplexes and G-quadruplex ligands: targets and tools in antiviral therapy. Nucleic Acids Res 2018, 46 (7), 3270-3283.

40. Mergny, J.-L.; Lacroix, L., UV Melting of G-Quadruplexes. Current Protocols in Nucleic Acid Chemistry 2009, 37 (1), 17.1.1-17.1.15.

41. Mergny, J.-L.; Lacroix, L., Analysis of Thermal Melting Curves. Oligonucleotides 2003, $13(6), 515-537$.

42. Zuffo, M.; Gandolfini, A.; Heddi, B.; Granzhan, A., Harnessing intrinsic fluorescence for typing of secondary structures of DNA. Nucleic Acids Res 2020, 48 (11), e61.

43. Gueddouda, N. M.; Hurtado, M. R.; Moreau, S.; Ronga, L.; Das, R. N.; Savrimoutou, S.; Rubio, S.; Marchand, A.; Mendoza, O.; Marchivie, M.; Elmi, L.; Chansavang, A.; Desplat, V.; Gabelica, V.; Bourdoncle, A.; Mergny, J. L.; Guillon, J., Design, Synthesis, and Evaluation of 2,9-Bis[(substituted-aminomethyl)phenyl]-1,10phenanthroline Derivatives as G-Quadruplex Ligands. ChemMedChem 2017, 12 (2), 146-160. 
44. Monchaud, D.; Allain, C.; Bertrand, H.; Smargiasso, N.; Rosu, F.; Gabelica, V.; De Cian, A.; Mergny, J. L.; Teulade-Fichou, M. P., Ligands playing musical chairs with Gquadruplex DNA: a rapid and simple displacement assay for identifying selective G-quadruplex binders. Biochimie 2008, 90 (8), 1207-23.

45. Mishra, S. K.; Jain, N.; Shankar, U.; Tawani, A.; Sharma, T. K.; Kumar, A., Characterization of highly conserved G-quadruplex motifs as potential drug targets in Streptococcus pneumoniae. Sci Rep 2019, 9 (1), 1791.

46. Bonnat, L.; Dautriche, M.; Saidi, T.; Revol-Cavalier, J.; Dejeu, J.; Defrancq, E.; Lavergne, T., Scaffold stabilization of a G-triplex and study of its interactions with Gquadruplex targeting ligands. Organic \& Biomolecular Chemistry 2019, 17 (38), 8726-8736.

47. De Rache, A.; Mergny, J. L., Assessment of selectivity of G-quadruplex ligands via an optimised FRET melting assay. Biochimie 2015, 115, 194-202.

48. Piazza, A.; Adrian, M.; Samazan, F.; Heddi, B.; Hamon, F.; Serero, A.; Lopes, J.; Teulade-Fichou, M. P.; Phan, A. T.; Nicolas, A., Short loop length and high thermal stability determine genomic instability induced by G-quadruplex-forming minisatellites. EMBO J 2015, 34 (12), 1718-34.

49. Chambers, V. S.; Marsico, G.; Boutell, J. M.; Di Antonio, M.; Smith, G. P.; Balasubramanian, S., High-throughput sequencing of DNA G-quadruplex structures in the human genome. Nat Biotechnol 2015, 33 (8), 877-81. 


\title{
FRET-MC: a fluorescence melting competition assay for studying G4 structures in vitro
}

\author{
Yu Luo ${ }^{1,2}$, Anton Granzhan ${ }^{1}$, Daniela Verga ${ }^{1^{*}}$ \& Jean-Louis Mergny ${ }^{2 *}$
}

1. Université Paris Saclay, CNRS UMR9187, INSERM U1196, Institut Curie, 91400 Orsay, France.

2. Laboratoire d'Optique et Biosciences, Ecole Polytechnique, CNRS, Inserm, Institut Polytechnique de Paris, 91128 Palaiseau, France.

* Authors to whom correspondence may be addressed: daniela.verga@curie.fr; jeanlouis.mergny@inserm.fr

\section{Supplementary information}

Tables S1-S4

Figures S1-S9 
Table S1. Training set of DNA sequences.

\begin{tabular}{|c|c|c|c|}
\hline Name & Sequence $\left(5^{\prime}-3^{\prime}\right)$ & $\begin{array}{l}\text { Reported } \\
\text { conformation }\end{array}$ & $\begin{array}{l}\text { PDB } \\
\text { entry }\end{array}$ \\
\hline F21T & FAM-GGGTTAGGGTTAGGGTTAGGG-TAMRA & - & - \\
\hline $46 \mathrm{AG}$ & $\begin{array}{l}\text { AGGGTTAGGGTTAGGGTTAGGGTTAGGGTTAGGGTTA } \\
\text { GGGTTAGGG }\end{array}$ & hybrid G4 & - \\
\hline Bcl2Mid & GGGCGCGGGAGGAATTGGGCGGG & hybrid G4 & $2 \mathrm{~F} 8 \mathrm{U}$ \\
\hline UpsB-Q3 & CAGGGTTAAGGGTATACATTTAGGGGTTAGGGTT & hybrid G4 & - \\
\hline 26TTA & TTAGGGTTAGGGTTAGGGTTAGGGTT & hybrid G4 & 2JPZ \\
\hline 25TGA & TAGGGTTAGGGTTAGGGTTAGGGTT & hybrid G4 & 2JSL \\
\hline 23TAG & TAGGGTTAGGGTTAGGGTTAGGG & hybrid G4 & $2 J S K$ \\
\hline 24TTA & TTAGGGTTAGGGTTAGGGTTAGGG & hybrid G4 & 2JSL \\
\hline VEGFR-17T & GGGTACCCGGGTGAGGTGCGGGGT & hybrid G4 & 5ZEV \\
\hline TP3-T6 & TGGGGTCCGAGGCGGGGCTTGGG & hybrid G4 & $6 \mathrm{AC7}$ \\
\hline chl1 & GGGTGGGGAAGGGGTGGGT & hybrid G4 & $2 \mathrm{KPR}$ \\
\hline UpsB-Q1 & CAGGGTTAAGGGTATAACTTTAGGGGTTAGGGTT & hybrid G4 & 5MTA \\
\hline LTR-III & GGGAGGCGTGGCCTGGGCGGGACTGGGG & hybrid G4 & $6 \mathrm{H} 1 \mathrm{~K}$ \\
\hline SP-PGQ-1 & GGGCAACTTGGCTGGGGTCTAGTTCCACGGGACGGG & hybrid G4 & - \\
\hline 26CEB & AAGGGTGGGTGTAAGTGTGGGTGGGT & parallel G4 & 2LPW \\
\hline $\begin{array}{l}\text { c-kit2- } \\
\text { T12T21 }\end{array}$ & CGGGCGGGCGCTAGGGAGGGT & parallel G4 & $2 \mathrm{KYP}$ \\
\hline KRAS-22RT & AGGGCGGTGTGGGAATAGGGAA & parallel G4 & $512 \mathrm{~V}$ \\
\hline Pu24T & TGAGGGTGGTGAGGGTGGGGAAGG & parallel G4 & $2 \mathrm{~A} 5 \mathrm{P}$ \\
\hline c-kit87up & AGGGAGGGCGCTGGGAGGAGGG & parallel G4 & $2 \mathrm{OMM}$ \\
\hline VEGF & CGGGGCGGGCCTTGGGCGGGGT & parallel G4 & $2 \mathrm{M} 27$ \\
\hline c-myc & TGAGGGTGGGTAGGGTGGGTAA & parallel G4 & 1XAV \\
\hline T95-2T & TTGGGTGGGTGGGTGGGT & parallel G4 & 2LK7 \\
\hline SP-PGQ-2 & GGGCTAGTGGGGGGAGGGGG & parallel G4 & - \\
\hline SP-PGQ-3 & GGGCTAATAGGGAGAGCAGGGACGGGG & parallel G4 & - \\
\hline PCNA G4 & CAGGGCGACGGGGGCGGGGCGGGGCG & parallel G4 & - \\
\hline TB-1 & TTGTGGTGGGTGGGTGGGT & parallel G4 & $2 \mathrm{M} 4 \mathrm{P}$ \\
\hline T2B-1 & TTGTTGGTGGGTGGGTGGGT & parallel G4 & - \\
\hline TB-3 & TTGGGTGTGGTGGGTGGGT & parallel G4 & - \\
\hline G15 & TTGGGGGGGGGGGGGGGT & parallel G4 & 2MB2 \\
\hline Myc1245 & TTGGGGAGGGTTTTAAGGGTGGGGAAT & parallel G4 & 6NEB \\
\hline AT11 & TGGTGGTGGTTGTTGTGGTGGTGGTGGT & parallel G4 & $2 \mathrm{~N} 3 \mathrm{M}$ \\
\hline LTR-IV & CTGGGCGGGACTGGGGAGTGGT & parallel G4 & $2 \mathrm{~N} 4 \mathrm{Y}$ \\
\hline hras-1 & TCGGGTTGCGGGCGCAGGGCACGGGCG & anti-parallel G4 & - \\
\hline TBA & GGTTGGTGTGGTTGG & anti-parallel G4 & 148D \\
\hline
\end{tabular}




\begin{tabular}{|c|c|c|c|}
\hline HIV-PRO-1 & TGGCCTGGGCGGGACTGGG & anti-parallel G4 & - \\
\hline 22CTA & AGGGCTAGGGCTAGGGCTAGGG & anti-parallel G4 & - \\
\hline Bm-U16 & TAGGTTAGGTTAGGTUAGG & anti-parallel G4 & - \\
\hline$c-k^{*}{ }^{\star}$ & GGCGAGGAGGGGCGTGGCCGGC & anti-parallel G4 & $6 \mathrm{GHO}$ \\
\hline Bom17 & GGTTAGGTTAGGTTAGG & anti-parallel G4 & - \\
\hline G4CT & GGGGCTGGGGCTGGGGCTGGGG & anti-parallel G4 & - \\
\hline 22GGG & GGGTTAGGGTTAGGGTTAGGGT & anti-parallel G4 & 2KF8 \\
\hline $19 w t$ & GGGGGAGGGGTACAGGGGTACAGGGG & anti-parallel G4 & $6 \mathrm{FTU}$ \\
\hline LWDLN 1 & GGGTTTGGGTTTTGGGAGGG & anti-parallel G4 & $5 \mathrm{~J} 05$ \\
\hline LWDLN 2 & GGGGTTGGGGTTTTGGGGAAGGGG & anti-parallel G4 & $2 \mathrm{M} 6 \mathrm{~W}$ \\
\hline LWDLN 3 & GGTTTGGTTTTGGTTGG & anti-parallel G4 & $5 \mathrm{~J} 4 \mathrm{~W}$ \\
\hline ss 3 & GTCGCCGGGCCAGTCGTCCATAC & single strand & - \\
\hline ss 4 & GTATGGACGACTGGCCCGGCGAC & single strand & - \\
\hline ss 6 & GACGTGTCGAAAGAGCTCCGATTA & single strand & - \\
\hline ss 7 & TAATCGGAGCTCTTTCGACACGTC & single strand & - \\
\hline RND1 & CTATACGAAAACCTTTTGTATCATT & single strand & - \\
\hline RND2 & AATGATACAAAAGGTTTTCGTATAG & single strand & - \\
\hline RND3 & TAACGTTTATAATGTAGTCTCATTA & single strand & - \\
\hline RND4 & TAATGAGACTACATTATAAACGTTA & single strand & - \\
\hline RND6 & GTTGTCATTGCCCCCGAATAATTCT & single strand & - \\
\hline RND7 & GCCTTGCGGAGGCATGCGTCATGCT & single strand & - \\
\hline RND8 & AGCATGACGCATGCCTCCGCAAGGC & single strand & - \\
\hline dT26 & TTTTTTTTTTTTTTTTTTTTTTTTTTT & single strand & - \\
\hline ds26 & CAATCGGATCGAATTCGATCCGATTG & duplex & - \\
\hline ds-lac & GAATTGTGAGCGCTCACAATTC & duplex & - \\
\hline Hairpin 1 & GGATTCTTGGATTTTCCAAGAATCC & duplex & - \\
\hline Hairpin 2 & TCGGTATTGTGTTTCACAATACCGA & duplex & - \\
\hline Hairpin 3 & AGGACGGTGTATTTTACACCGTCCT & duplex & - \\
\hline \multirow[t]{2}{*}{ d34 } & GTCGCCGGGCCAGTCGTCCATAC & & - \\
\hline & GTATGGACGACTGGCCCGGCGAC & duplex & - \\
\hline \multirow[t]{2}{*}{ d67 } & GACGTGTCGAAAGAGCTCCGATTA & & - \\
\hline & TAATCGGAGCTCTTTCGACACGTC & duplex & - \\
\hline \multirow[t]{2}{*}{ RND34 } & TAACGTTTATAATGTAGTCTCATTA & & - \\
\hline & TAATGAGACTACATTATAAACGTTA & duplex & - \\
\hline \multirow[t]{2}{*}{ RND78 } & AGAATTATTCGGGGGCAATGACAAC & & - \\
\hline & GTTGTCATTGCCCCCGAATAATTCT & duplex & - \\
\hline
\end{tabular}


Table S2. Tm of some competitors collected by UV-melting ${ }^{a}$

\begin{tabular}{|c|c|c|c|}
\hline Name & $\mathrm{Tm} /{ }^{\circ} \mathrm{C}$ & Note & Reported conformation \\
\hline $\mathrm{F} 21 \mathrm{~T}$ & $58.1^{a}$ & Probe & - \\
\hline UpsB-Q3 & 39.4 & False negative & hybrid G4 \\
\hline SP-PGQ-1 ${ }^{b}$ & 46.8 & Not a G4; rather a duplex & hybrid G4 \\
\hline KRAS-22RT & 32.8 & False negative & parallel G4 \\
\hline SP-PGQ-3 & 34.7 & False negative & parallel G4 \\
\hline TBA & 42.1 & False negative & anti-parallel G4 \\
\hline $\mathrm{Bm}-\mathrm{U} 16$ & 26.8 & False negative & anti-parallel G4 \\
\hline LWDLN3 & 33.0 & False negative & anti-parallel G4 \\
\hline Bom17 & 30.3 & False negative & anti-parallel G4 \\
\hline $46 A G$ & 43.3 & Positive control $c$ & hybrid G4 \\
\hline Bcl2Mid & 52.0 & Positive control $c$ & hybrid G4 \\
\hline 25TGA & 47.3 & Positive control $c$ & hybrid G4 \\
\hline Chl1 & 62.7 & Positive control $c$ & hybrid G4 \\
\hline LTR-III & 46.5 & Positive control $c$ & hybrid G4 \\
\hline c-kit-T12T2 & 46.2 & Positive control $c$ & parallel G4 \\
\hline Pu24T & 74.1 & Positive control $c$ & parallel G4 \\
\hline c-kit87up & 48.7 & Positive control $c$ & parallel G4 \\
\hline VEGF & 66.5 & Positive control $c$ & parallel G4 \\
\hline T95-2T & 81.4 & Positive control $c$ & parallel G4 \\
\hline 22CTA & 45.5 & - & anti-parallel G4 \\
\hline $\mathrm{G} 4 \mathrm{CT}$ & 69.5 & - & anti-parallel G4 \\
\hline $22 G G G$ & 54.4 & - & anti-parallel G4 \\
\hline $19 w t$ & 80.9 & - & anti-parallel G4 \\
\hline LWDLN1 & 45.1 & - & anti-parallel G4 \\
\hline ds26 & 76.3 & - & duplex \\
\hline ds-lac & 70.1 & - & duplex \\
\hline Hairpin1 & 71.4 & - & duplex \\
\hline Hairpin2 & 74.9 & - & duplex \\
\hline d34 & 78.8 & - & duplex \\
\hline
\end{tabular}

${ }^{a} \mathrm{Tm}$ of F21T was measured by FRET-melting, Tm of reported G4 sequences and duplex were collected at $295 \mathrm{~nm}$ and $260 \mathrm{~nm}$, respectively.

${ }^{b} \mathrm{Tm}$ of SP-PGQ-1 calculated by UV-melting curve at $260 \mathrm{~nm}$.

${ }^{c}$ Positive controls are G4-forming sequences which give an $\mathrm{S}$ value close to 0 . 
Table S3. Tm and $S$ Factor average of some sequences in the training set

\begin{tabular}{|c|c|c|}
\hline Name & $\mathrm{Tm}^{\circ}{ }^{\circ} \mathbf{C}^{a}$ & S Factor average $^{b}$ \\
\hline 46AG & 43.3 & 0.49 \\
\hline c-kit2-T12T12 & 46.2 & 0.40 \\
\hline 25TAG & 47.3 & 0.60 \\
\hline Bcl2Mid & 52.0 & 0.62 \\
\hline 22GGG & 54.4 & 0.50 \\
\hline G4CT & 69.5 & 0.35 \\
\hline Pu24T & 74.1 & 0.23 \\
\hline 19wt & 80.9 & 0.22 \\
\hline T95-2T & 81.4 & 0.26 \\
\hline
\end{tabular}

${ }^{a}$ Tm were collected at $295 \mathrm{~nm}$.

${ }^{b} S$ Factor averages were determined by the $S$ of $0.2 / 0.6 / 1 / 2 \mu \mathrm{M}$ (1x to $10 \mathrm{x}$ molar excess, as compared to F21T) competitor concentrations. 
Table S4. Testing set of DNA sequences.

\begin{tabular}{|l|l|c|l|}
\hline Name & Sequence $\left(5^{\prime}\right.$-3') & G4-Hunter score & Location (Human hg19) \\
\hline F21T & $\begin{array}{l}\text { FAM-GGGTTAGGGTTAGGGTTAGGG- } \\
\text { TAMRA }\end{array}$ & - & - \\
\hline UN1 & $\begin{array}{l}\text { CGGGCAGGGAGGGCGGCTGTGCGGG } \\
\text { GC }\end{array}$ & 1.59 & chr3: 196045150-196045176 \\
\hline UN2 & $\begin{array}{l}\text { TGGGGCGGGGGAAGAGGGGGCGGGG } \\
\text { T }\end{array}$ & 2.73 & chr8: 57124113-57124138 \\
\hline UN3 & CGGGAAGGGGCGGGCGCAATGGGC & 1.62 & chr17: 62915412-62915435 \\
\hline UN4 & TGGGAGGCGGAGGTGGGCAGGTTGCT & 1.23 & chr17: 16258771-16258791 \\
\hline UN5 & $\begin{array}{l}\text { GTGCTGGGGCGCCCACTTCGGGGTGG } \\
\text { TGC }\end{array}$ & 0.90 & chr11: 364605-364633 \\
\hline UN6 & $\begin{array}{l}\text { AGTTGGTAGGCTGAGGCGGGAGGATT } \\
\text { GC }\end{array}$ & 0.89 & chr5: 64905453-64905472 \\
\hline UN7 & $\begin{array}{l}\text { AGGGCCGGGAGAGGGATCCGCCATAT } \\
\text { TGGAGCTGGGGC }\end{array}$ & 0.94 & chr14: $36278253-36278290$ \\
\hline UN8 & $\begin{array}{l}\text { AGGAAGCTGGGGTAGGAGAATTGCTTG } \\
\text { A }\end{array}$ & 0.93 & chr12: 57876780-57876802 \\
\hline
\end{tabular}


A

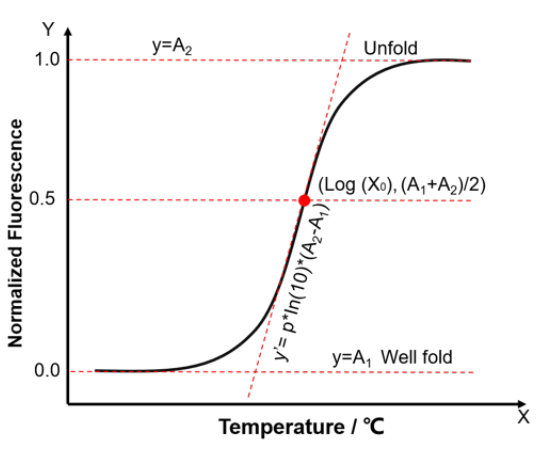

C

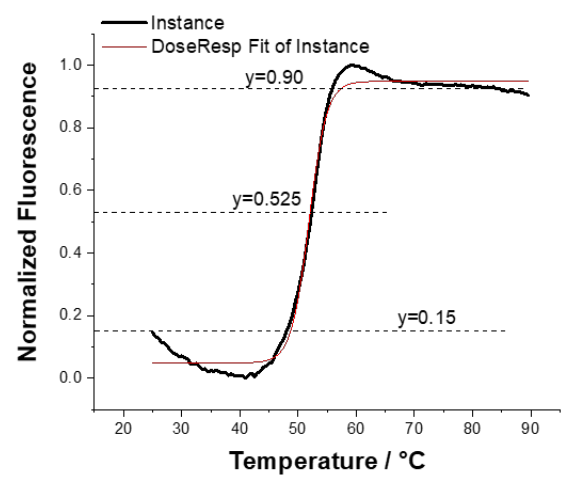

B

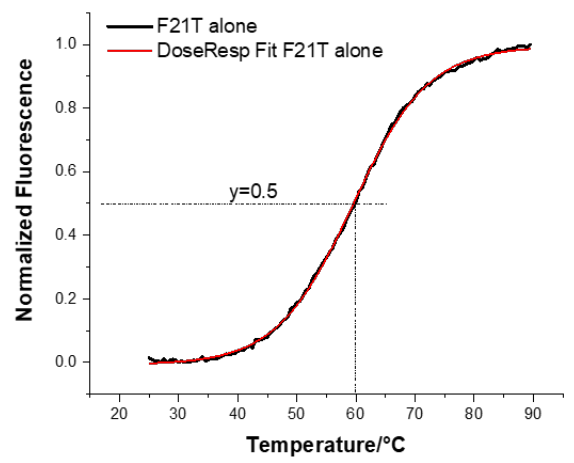

Fig. S1. Different methods to calculate Tm. (A) Sample curve of DoseResp. (B) Two methods to calculate Tm of F21T alone. (C) Example of an atypical FRET-melting curve, for which accurate Tm determination is more difficult. 


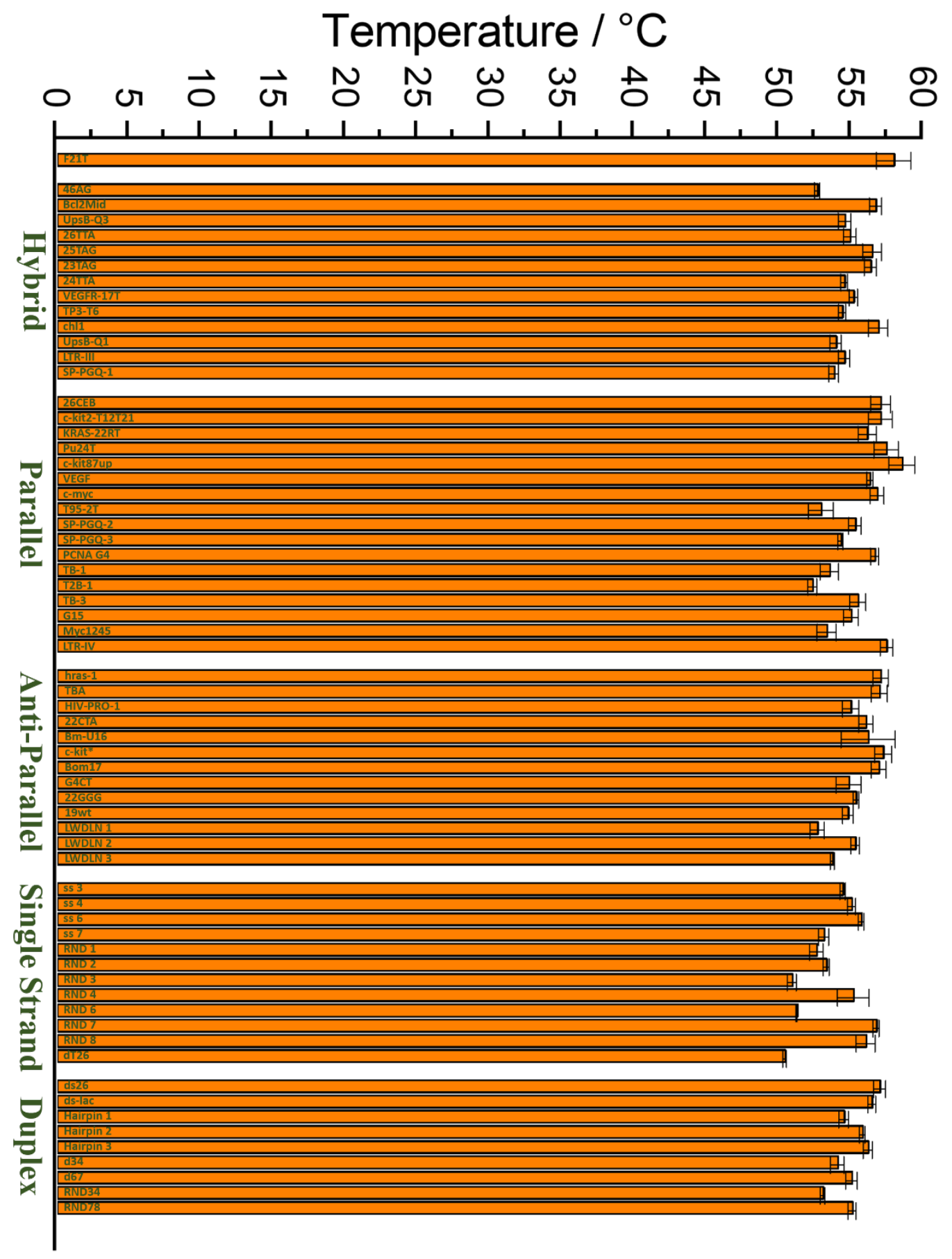

Fig. S2. FRET Tm of $0.2 \mu \mathrm{M}$ F21T alone (top) or in the presence of various competitors.

All competitors were tested at $3 \mu \mathrm{M}$ strand concentration. 
A

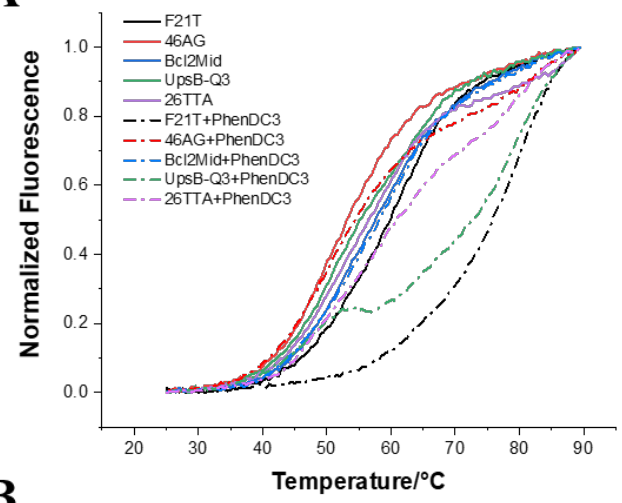

B

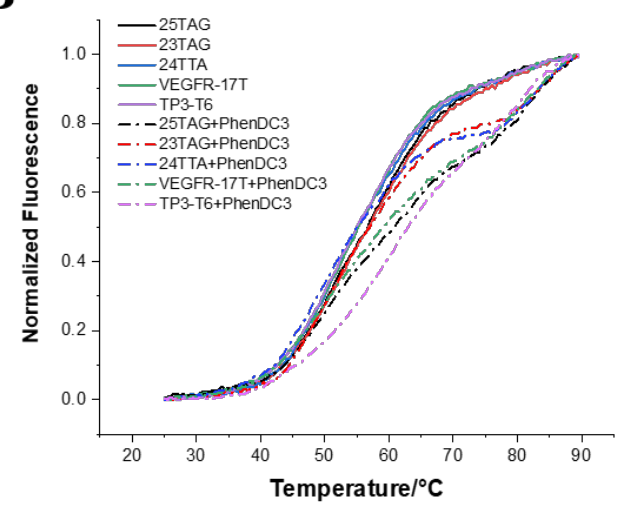

C

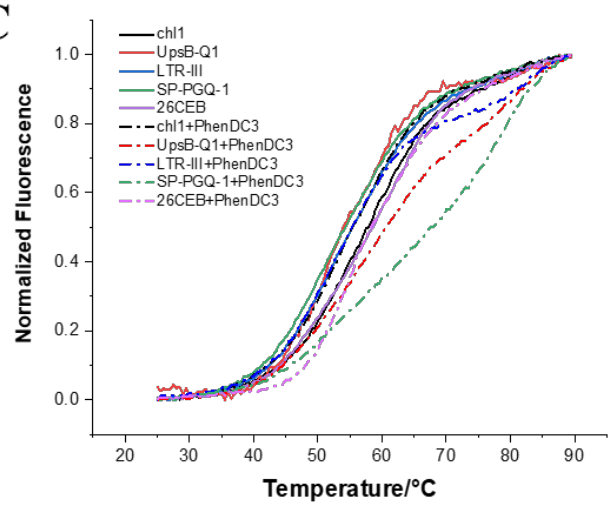

D

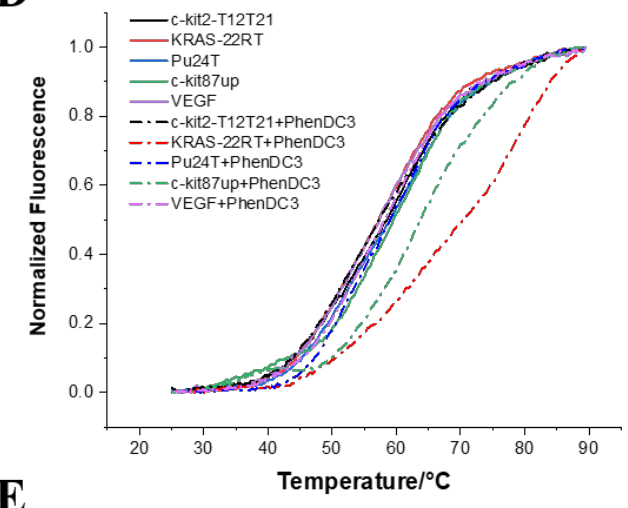

$\mathbf{E}$

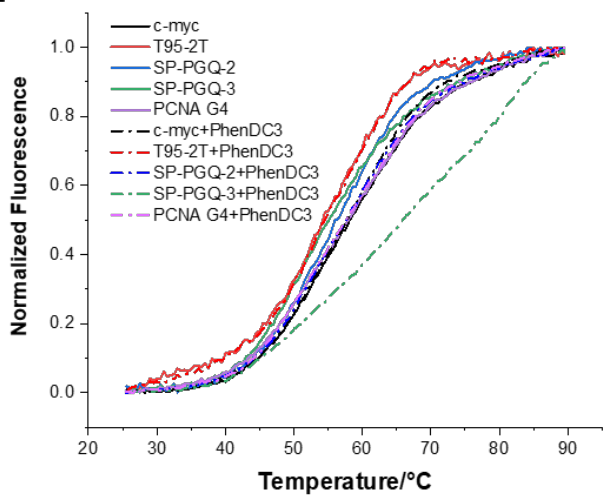

$\mathbf{F}$

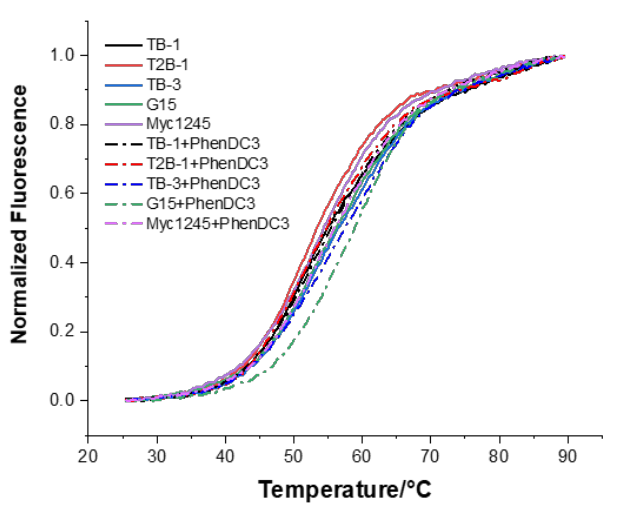

Fig. S3. Normalized FRET-melting curves of $0.2 \mu \mathrm{M}$ F21T in the presence of various competitors ( $3 \mu \mathrm{M}$ strand concentration) used in the training set, with or without $0.4 \mu \mathrm{M}$ PhenDC3. Samples were annealed and measured in $10 \mathrm{mM} \mathrm{KCl}, 90 \mathrm{mM} \mathrm{LiCl}, 10 \mathrm{mM}$ lithium cacodylate $\mathrm{pH} 7.2$ buffer. 

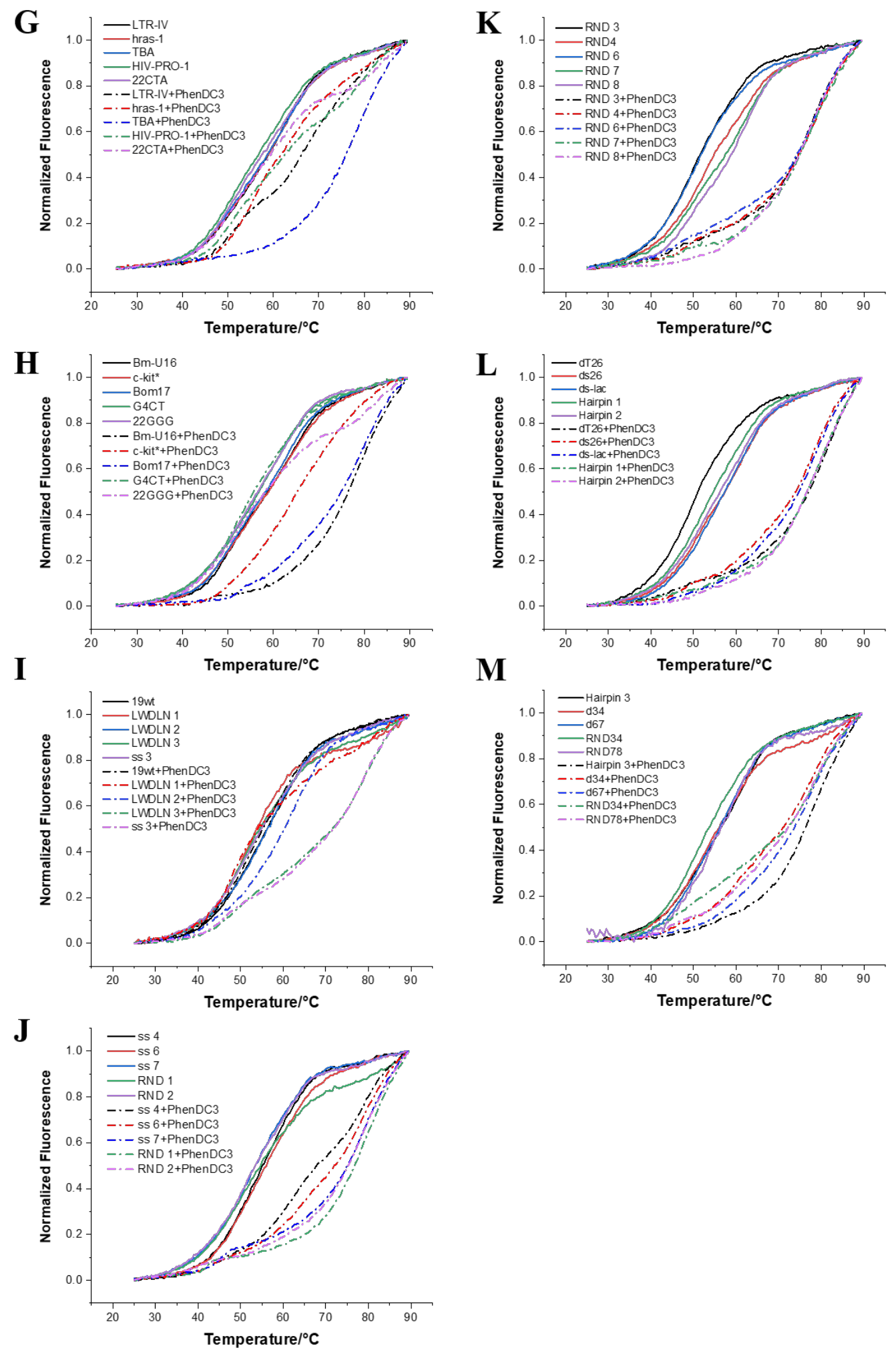

Fig. S3, continued. 
A

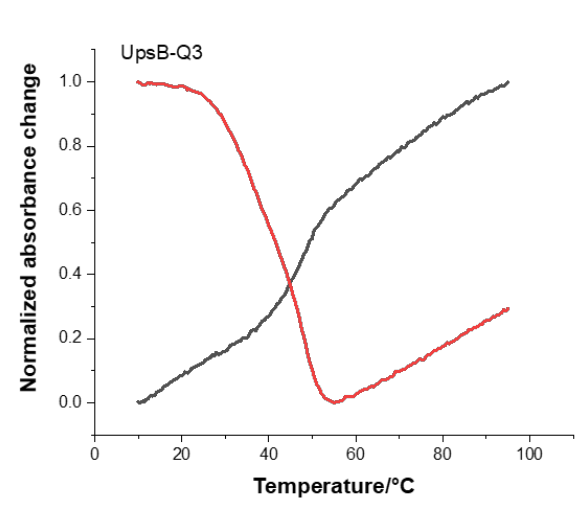

B

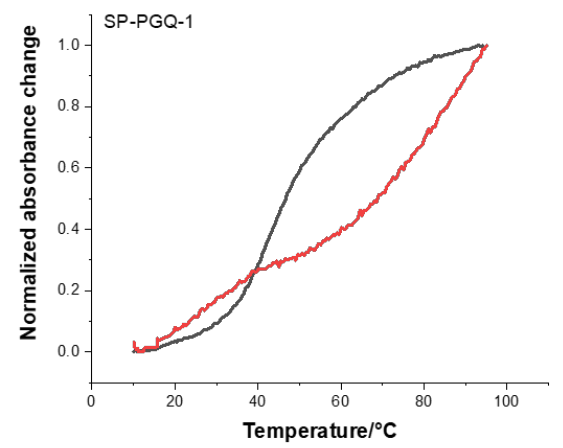

C

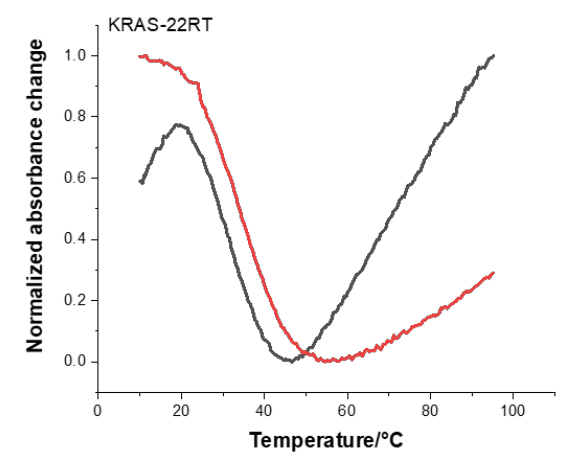

D

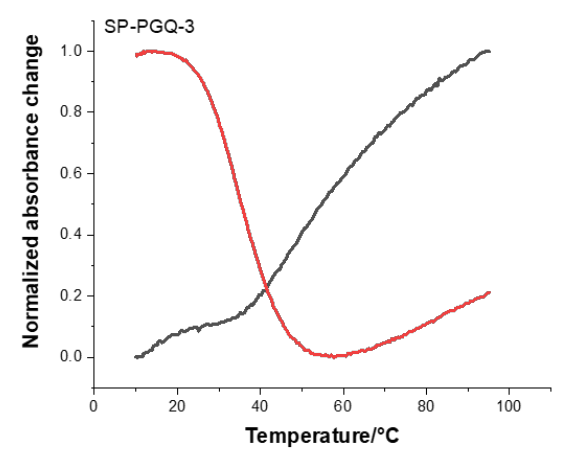

$\mathbf{E}$
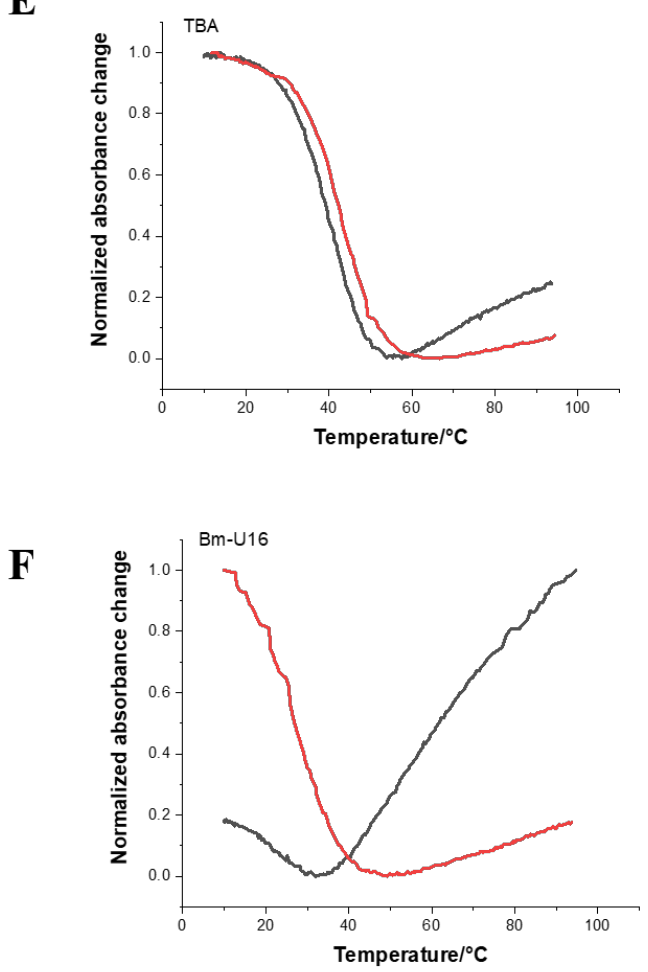

G

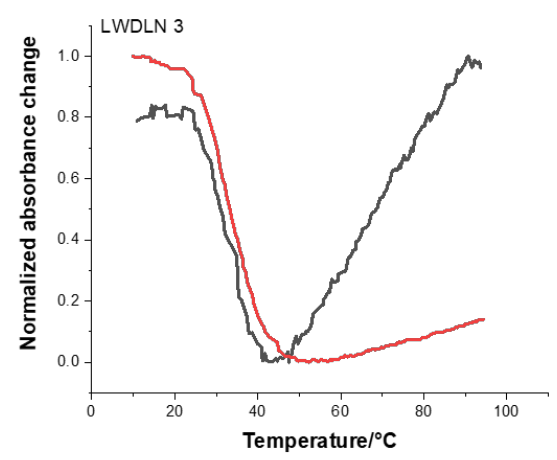

$\mathbf{H}$

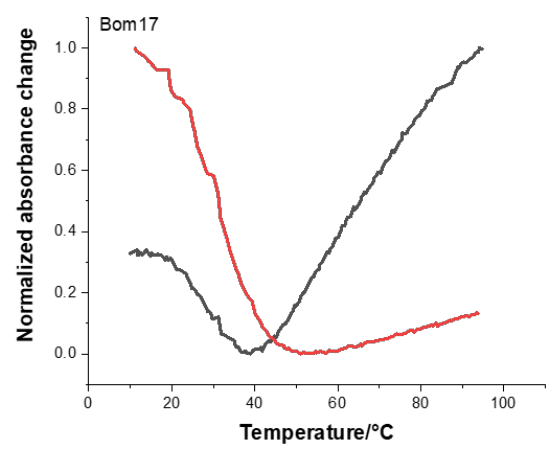

Fig. S4. Normalized UV-melting curves for some sequences of the training set. All data collected in 10-95 ${ }^{\circ} \mathrm{C}$, monitored at $260 \mathrm{~nm}$ (Blank lines) and $295 \mathrm{~nm}$ (Red lines). 
I

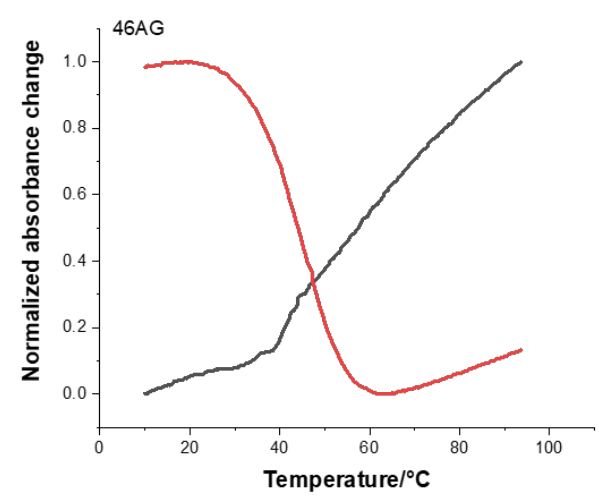

J

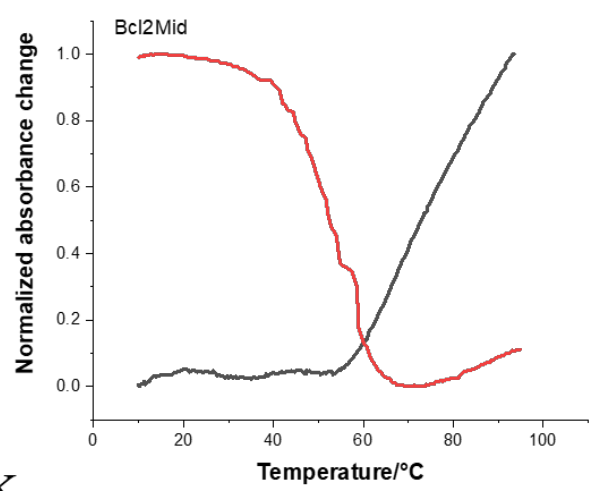

K

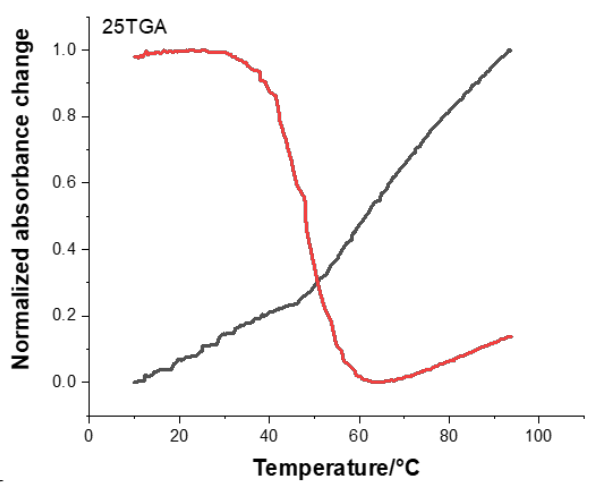

L

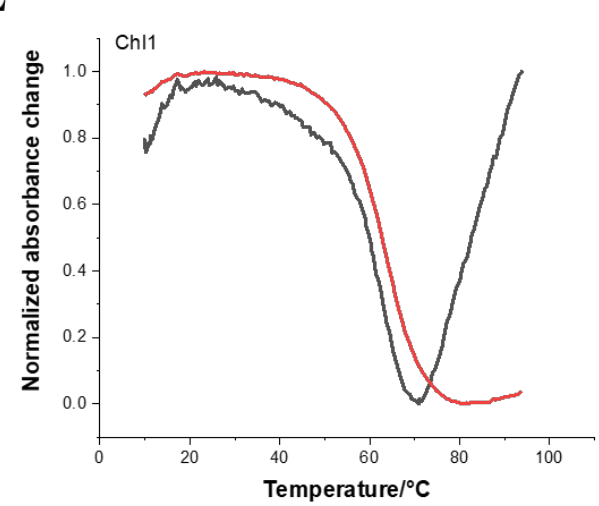

M

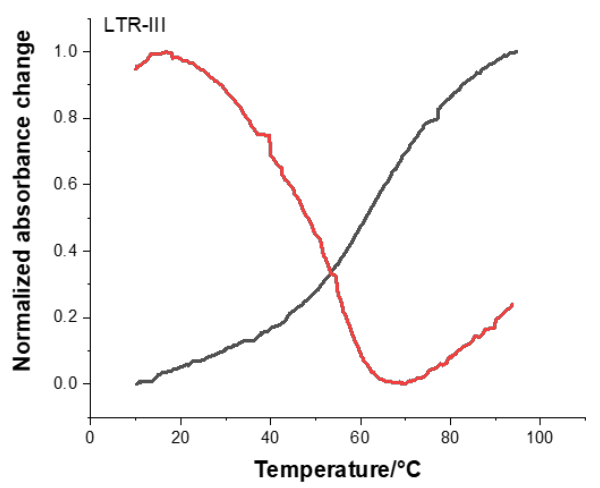

N

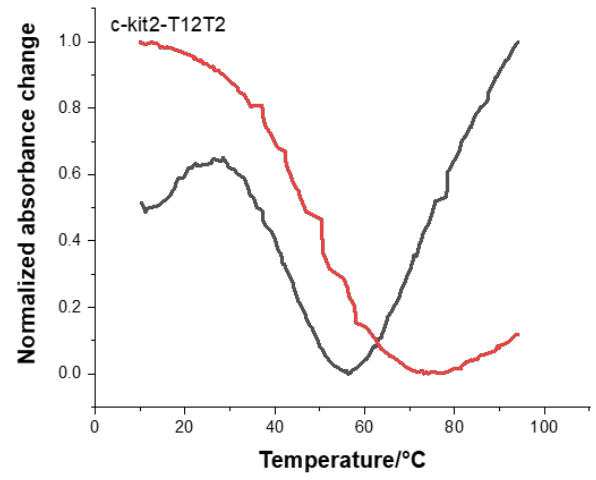

O

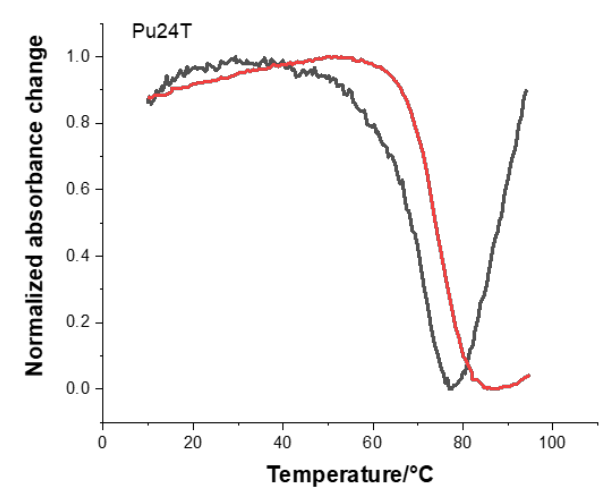

$\mathbf{P}$

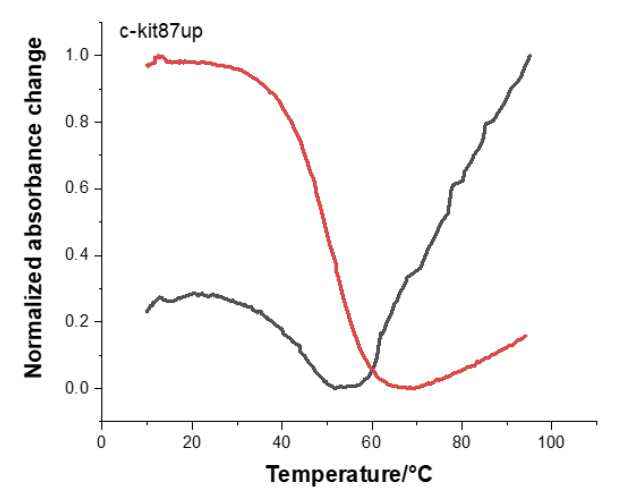

Fig. S4. continued. 

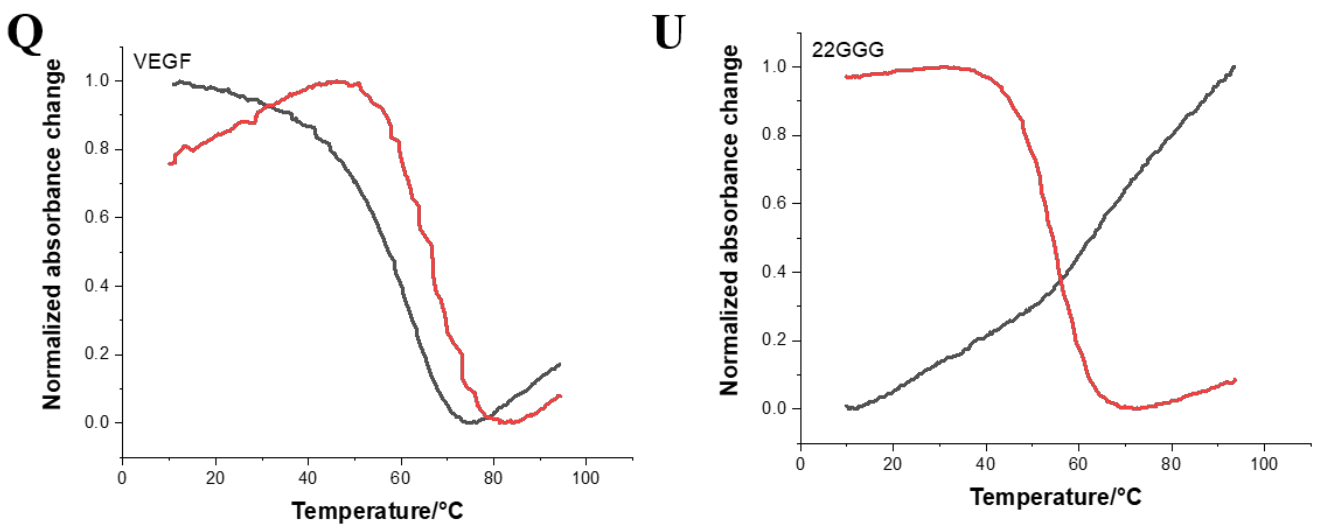

$\mathbf{R}$

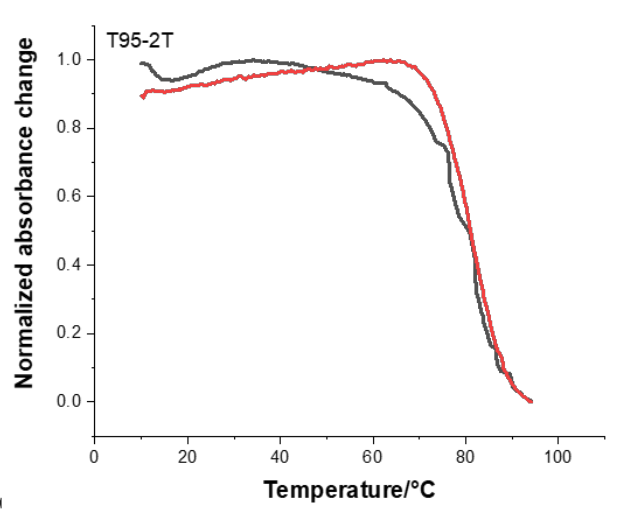

V

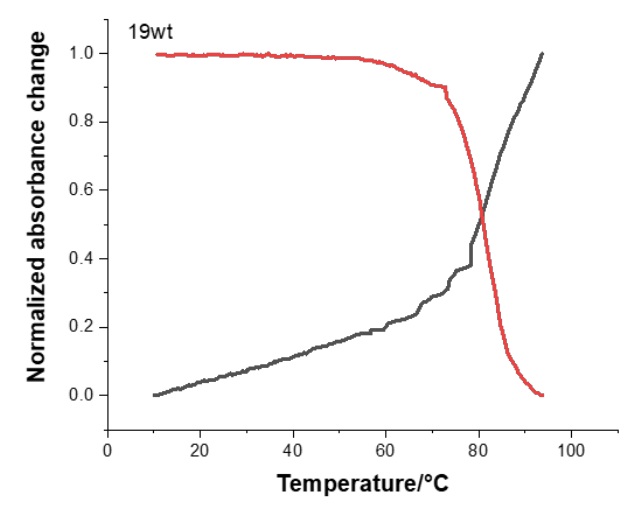

$\mathbf{S}$

W
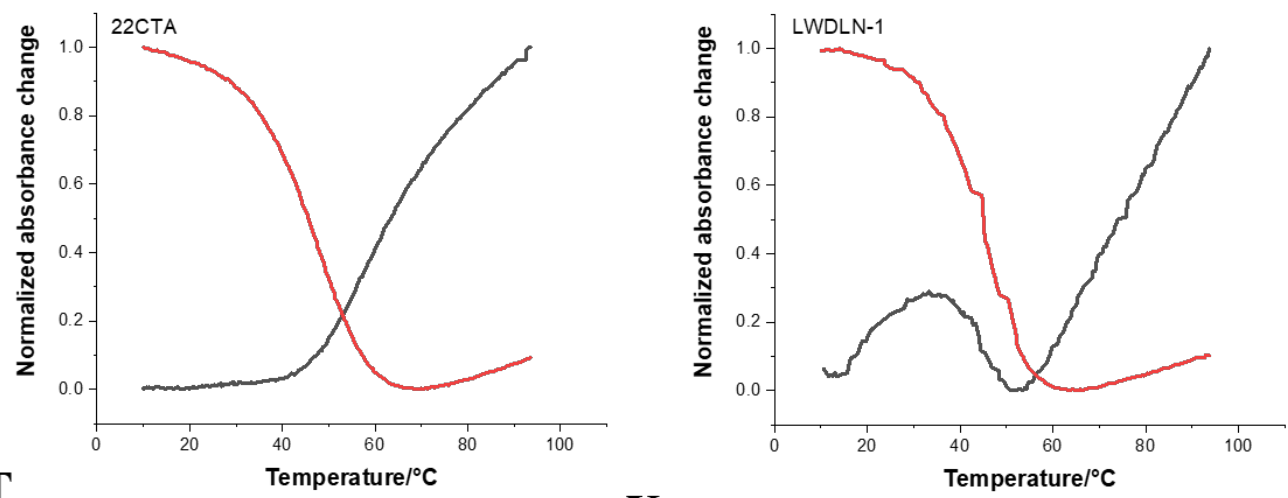

$\mathbf{X}$
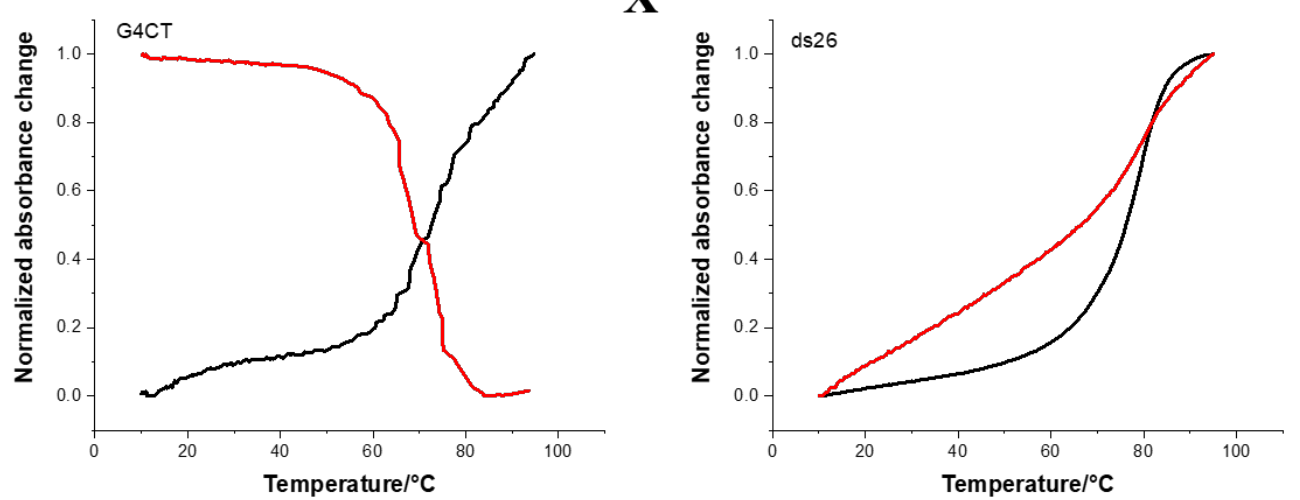

Fig. S4. continued. 
Y

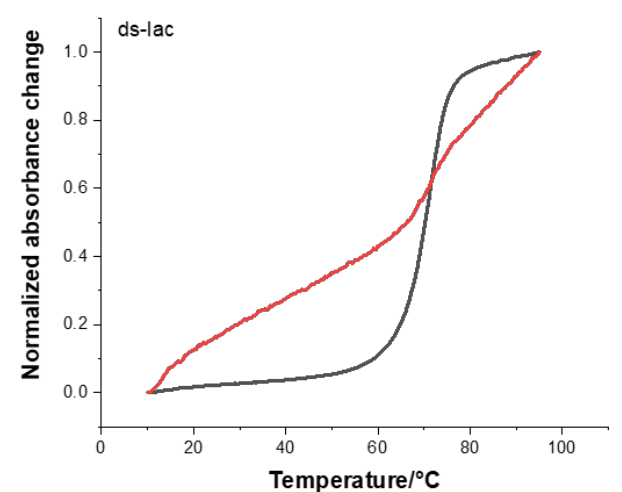

Z

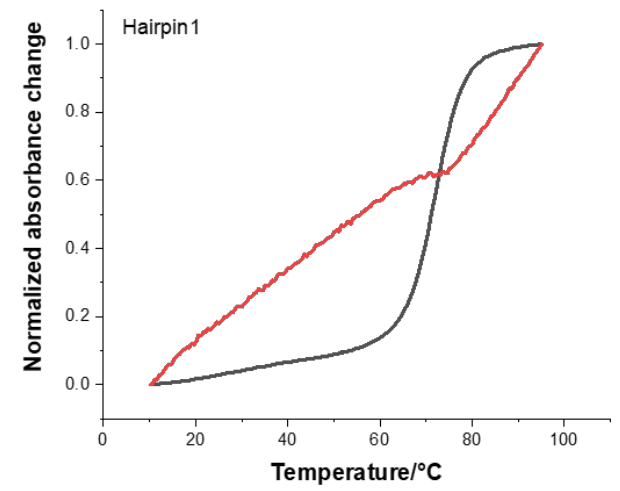

AA

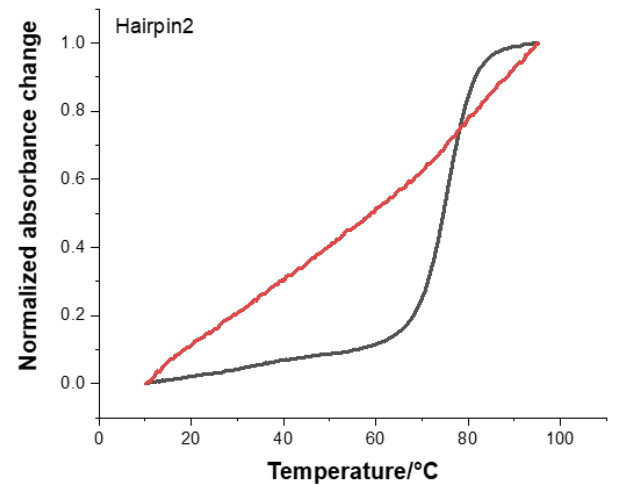

AB

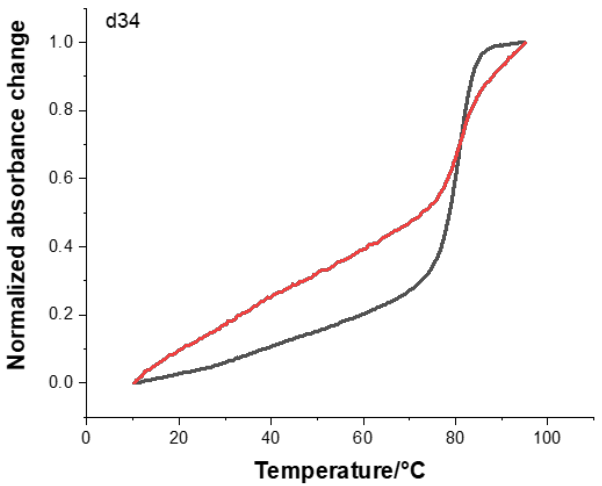

Fig. S4. continued. 

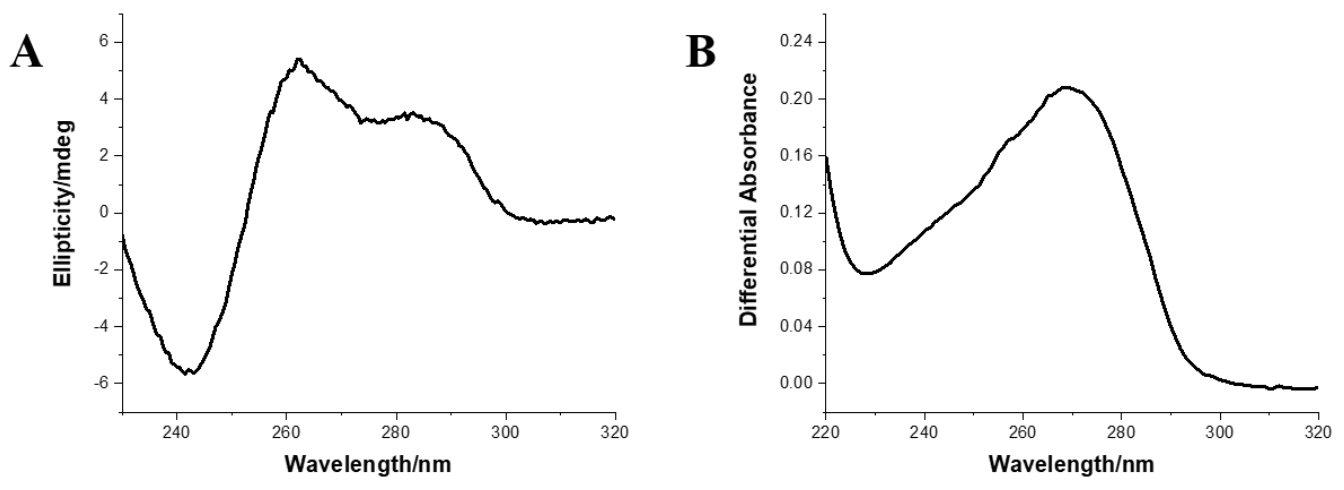

Fig. S5. CD (A) and thermal differential absorbance (TDS) (B) spectra for $3 \mu \mathrm{M}$ SP-PGQ-1 in the FRET buffer. 


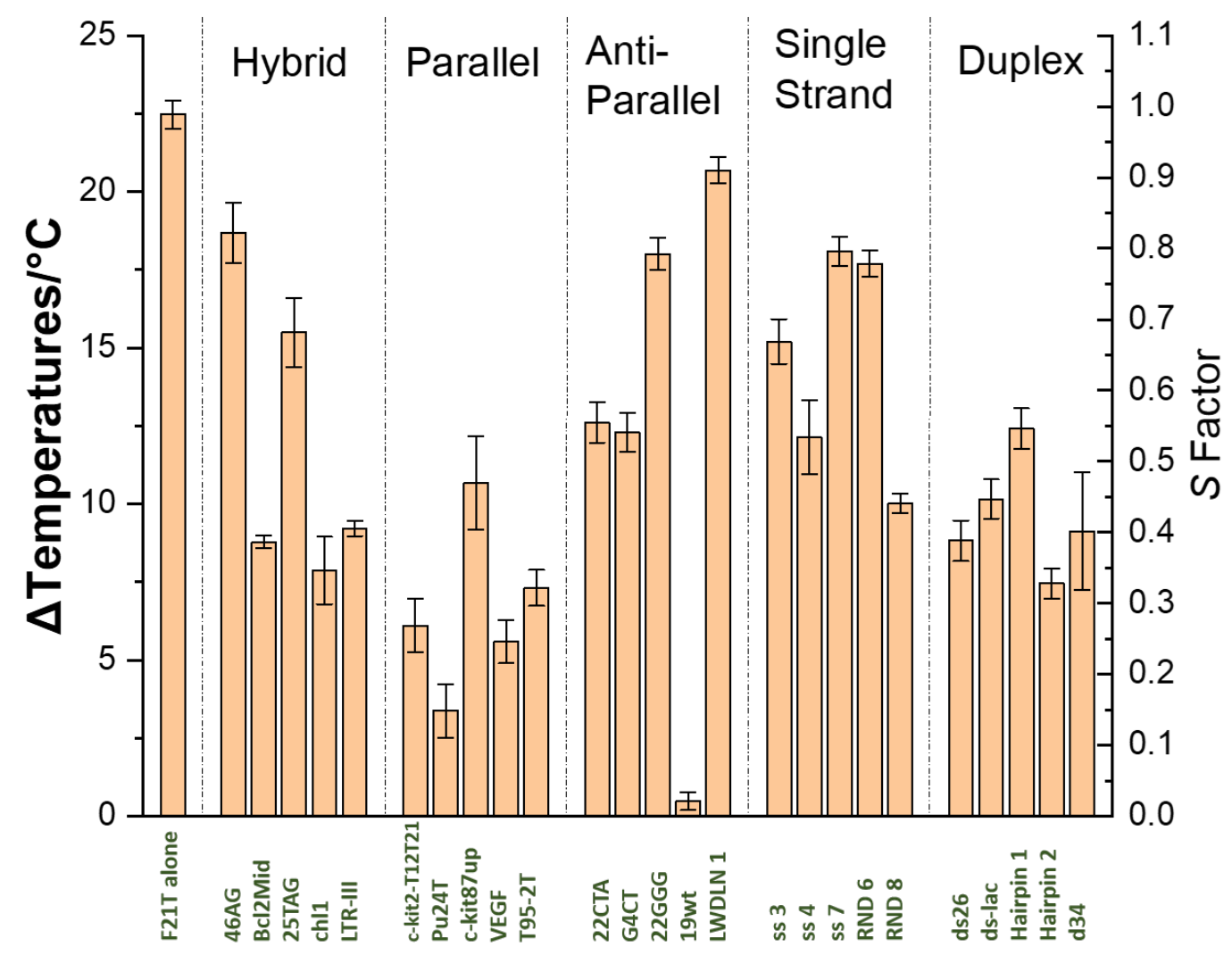

Fig. S6. $\Delta$ Tm induced by $0.4 \mu M$ TMPyP4 on $0.2 \mu M$ F21T, alone or in the presence of 3 $\boldsymbol{\mu M}$ competitors. The $S$ Factor is also provided on the right Y-axis. Samples were annealed and measured in $10 \mathrm{mM} \mathrm{KCl}, 90 \mathrm{mM} \mathrm{LiCl}, 10 \mathrm{mM}$ lithium cacodylate $\mathrm{pH} 7.2$ buffer. 


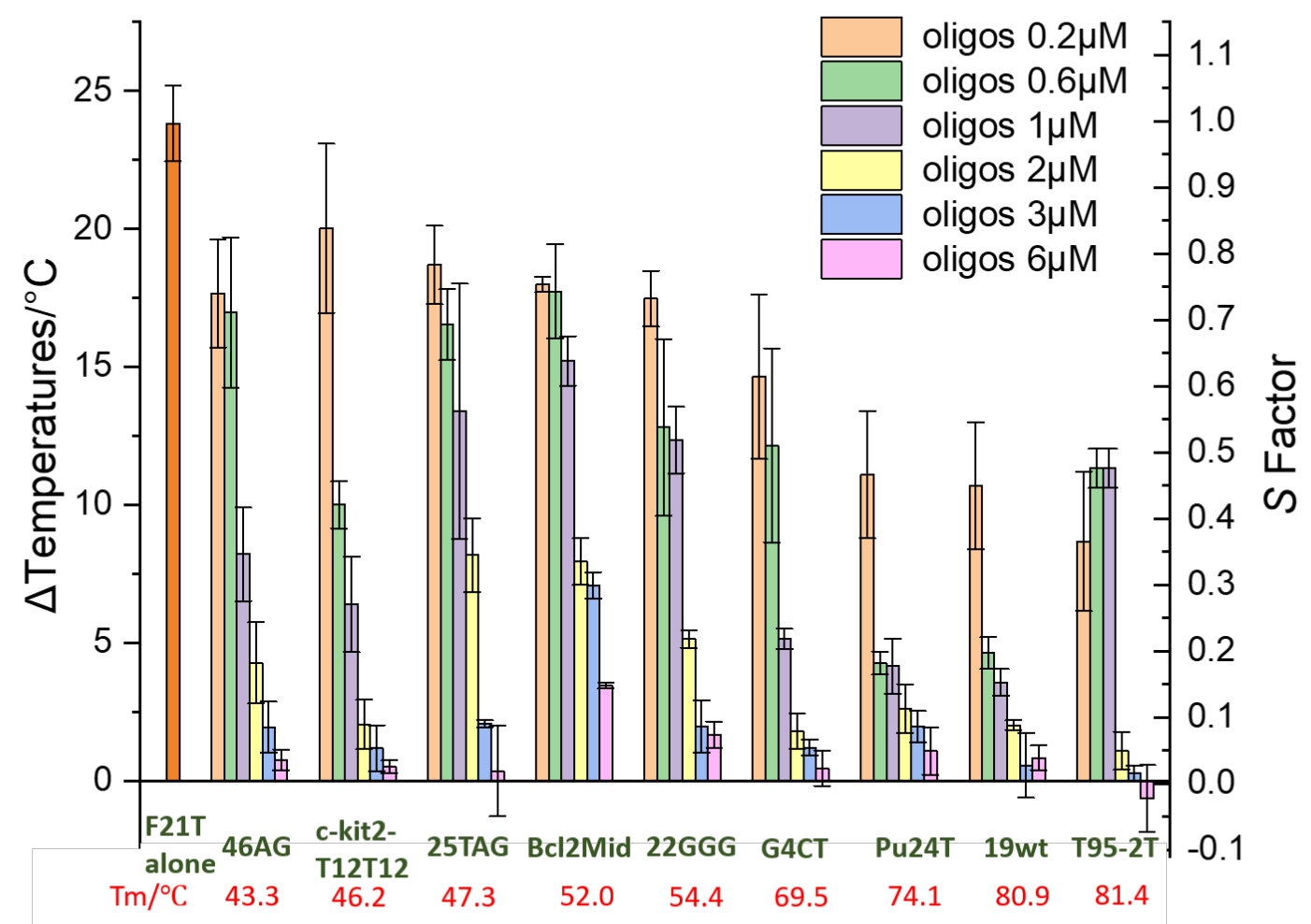

Fig. S7. $\Delta$ Tm induced by $0.4 \mu \mathrm{M}$ PhenDC3 on $0.2 \mu \mathrm{M}$ F21T, alone or in the presence of 0.2 / 0.6 / 1 / 2 / 3 / $6 \mu$ M competitors ( $1 \times$ to 30x molar excess, as compared to F21T). The $S$ Factor is also provided on the right Y-axis. Samples were annealed and measured in $10 \mathrm{mM}$ $\mathrm{KCl}, 90 \mathrm{mM} \mathrm{LiCl}, 10 \mathrm{mM}$ lithium cacodylate $\mathrm{pH} 7.2$ buffer. The Tm for each quadruplex are shown in red below; sequences were ranked from left (lowest $\mathrm{Tm}$ ) to right (highest $\mathrm{Tm}$ ). 
A Low $\operatorname{Tm}\left(<50^{\circ} \mathrm{C}\right)$ group

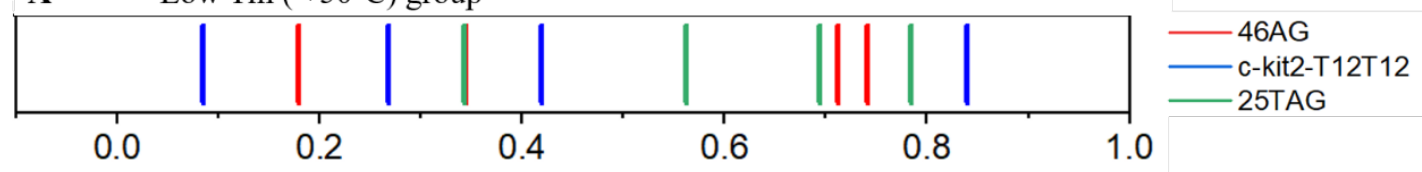

B $\quad$ Middle $\operatorname{Tm}\left(50-70^{\circ} \mathrm{C}\right)$ group

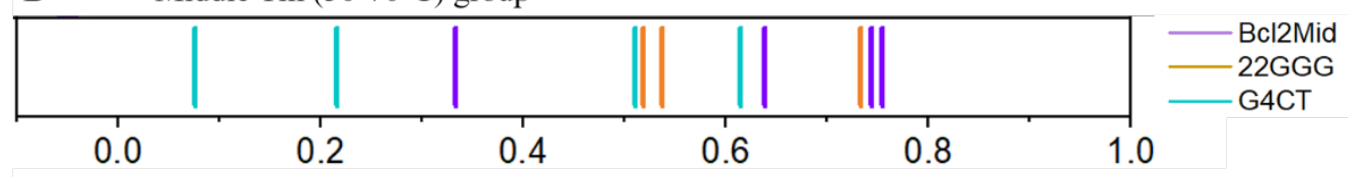

C High $\operatorname{Tm}\left(>70^{\circ} \mathrm{C}\right)$ group

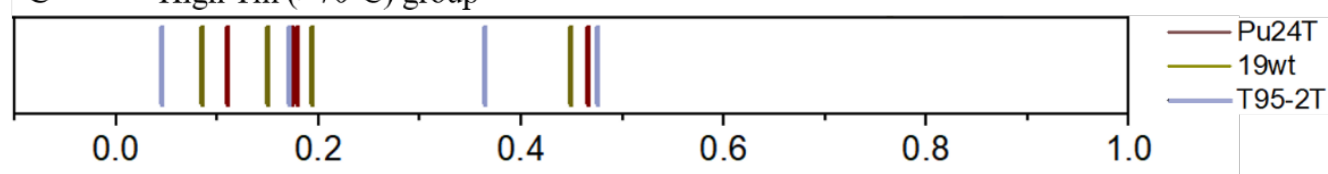

\section{Distribution of $S$ Factor}

Fig. S8. Distribution of S-factor values for $0.2 / 0.6 / 1 / 2 \mu \mathrm{M}$ competitor concentrations ( $1 \mathrm{x}$ to 10x molar excess, as compared to F21T). Oligonucleotides are grouped based on Tm: low Tm $\left(<50 \mathrm{C}^{\circ}\right)(\mathbf{A})$, middle $\operatorname{Tm}\left(50-70 \mathrm{C}^{\circ}\right)(\mathbf{B})$ and high $\operatorname{Tm}\left(>70^{\circ} \mathrm{C}\right)(\mathbf{C})$. The $4 S$ values shown for each competitor are depicted with the same color; the lowest $S$ value (leftmost vertical bar) corresponds to the highest $(2 \mu \mathrm{M})$ concentration while the highest $S$ value (rightmost bar) corresponds to the lowest $(0.2 \mu \mathrm{M})$ concentration 
A
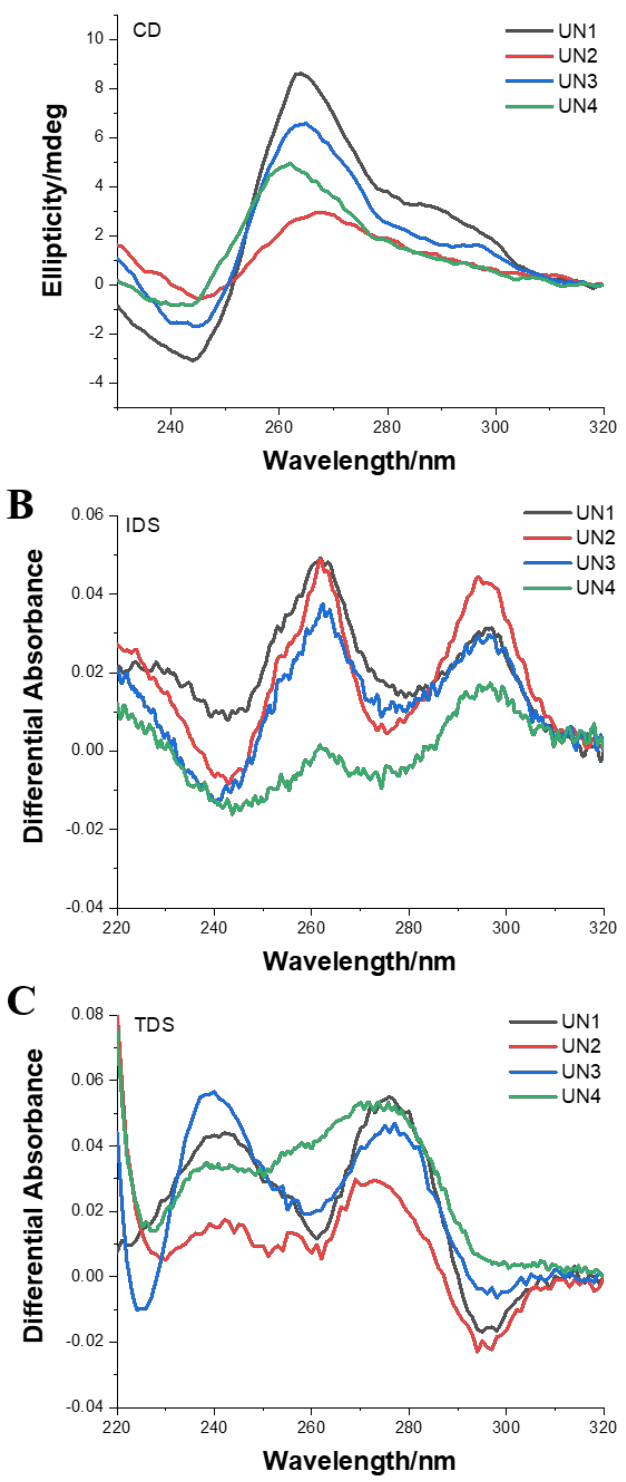

D

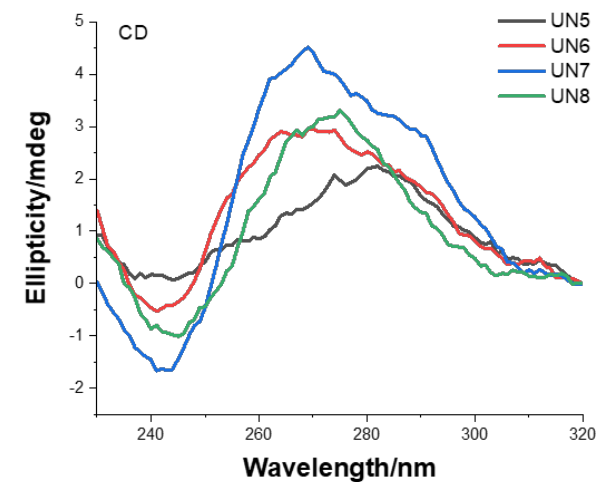

$\mathbf{E}$

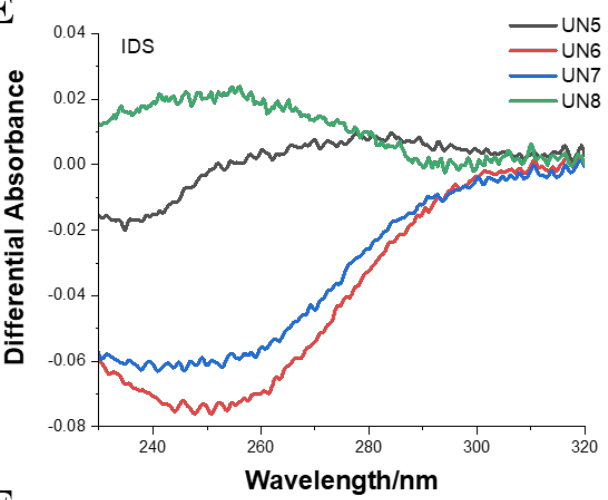

F

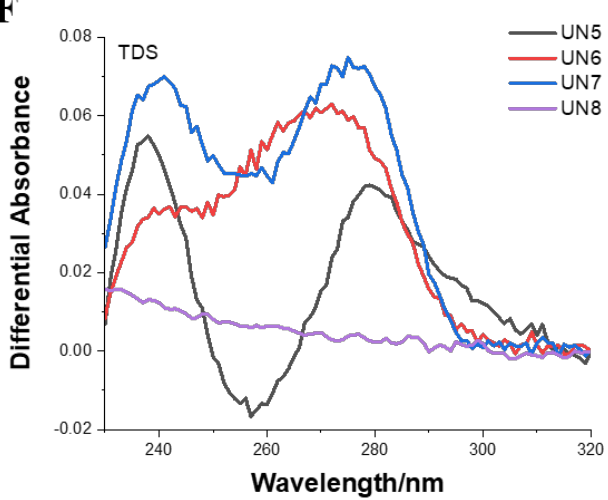

Fig. S9. CD, IDS, and TDS spectra of testing set. Panels A, B, C correspond to positive sequences (absolute $S$ values $<0.1$ ) and panels $\mathbf{D}, \mathbf{E}, \mathbf{F}$ to negative sequences, unable to compete $(\mathrm{S}>0.8)$. From top to bottom: CD spectra, IDS spectra, and TDS spectra. 